\title{
Motion Compensation using Principal Component Analysis and Projection onto Dipole Fields for Abdominal Magnetic Resonance Thermometry
}

\section{by}

Jeremy Hui-Min Tan

A thesis submitted in conformity with the requirements for the degree of Master of Health Science - Clinical Engineering

Institute of Biomaterials and Biomedical Engineering University of Toronto

(C) Copyright by Jeremy Tan 2016 


\title{
Motion Compensation using Principal Component Analysis and Projection onto Dipole Fields for Abdominal Magnetic Resonance Thermometry
}

\author{
Jeremy Tan \\ Master of Health Science - Clinical Engineering \\ Institute of Biomaterials and Biomedical Engineering \\ University of Toronto
}

2016

\begin{abstract}
High-intensity focused ultrasound is a non-invasive heat-based therapy, which is often guided by magnetic resonance (MR) thermometry. MR thermometry provides accurate temperature measurement at the target and in surrounding tissue. This is critical to ensure that sufficient thermal dose is achieved and to monitor for collateral damage. Unfortunately, MR thermometry is severely compromised by motion and susceptibility artifacts that are caused by respiration (periodic motion) and peristalsis (aperiodic motion). A hybrid method has been designed to correct these artifacts using principal component analysis as a multi-baseline method, and projection onto dipole fields as a near-referenceless approach. In vivo measurements in porcine and human kidneys, achieved a temperature stability and precision of $0.85{ }^{\circ} \mathrm{C}$ and $1.00{ }^{\circ} \mathrm{C}$, respectively, without the need for any user-interaction or supplementary tracking tools. This is about twice as accurate as the leading hybrid method, which scored $1.97{ }^{\circ} \mathrm{C}$ and $2.22{ }^{\circ} \mathrm{C}$, respectively, on the same data.
\end{abstract}




\section{Acknowledgments}

I must thank my supervisors, Dr. James Drake and Dr. Adam Waspe, for their endless encouragement and tireless support. They have given me a world of opportunities I could not have imagined for myself at the start of this program. They have also provided incredibly valuable insight in every aspect of the research process. I have been so fortunate to have them as role models and I will strive to mimic their unbelievably humble and humourous character.

I also must thank my committee members, Dr. Christopher Macgowan and Dr. John Sled, for their patience, acumen, and support. I can only hope to one day be as knowledgeable and adept as they are in this wonderful field.

I also received an incredible amount of support from Dr. Charles Mougenot and Dr. Samuel Pichardo. Every time I spoke with them I was amazed and became even more fascinated with this project.

The entire lab has also been incredibly helpful and supportive. This work would not have been possible without the support from Karolina Piorkowska and Steven Engler.

My friends and family have also been an invaluable source of support throughout this program. 


\section{Table of Contents}

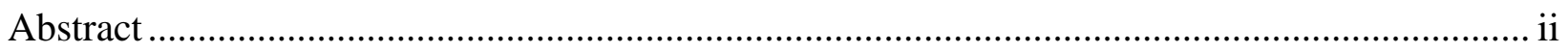

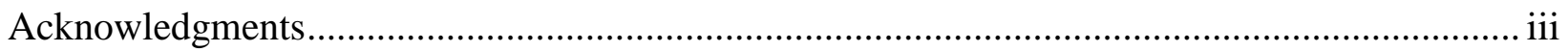

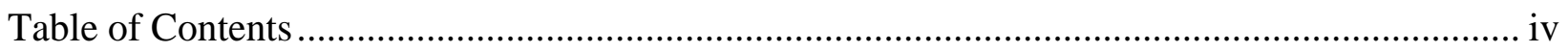

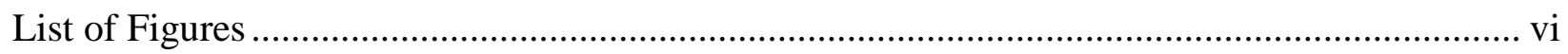

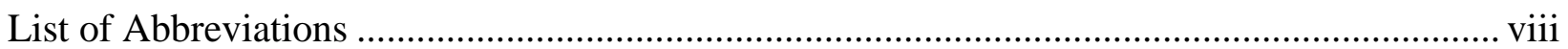

Chapter 1 Magnetic Resonance Thermometry for Monitoring Abdominal High-Intensity Focused Ultrasound Procedures ......................................................................................... 1

1.1. Introduction:

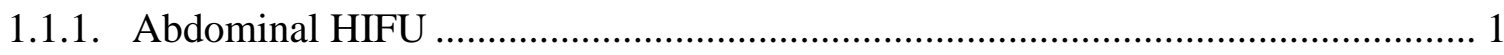

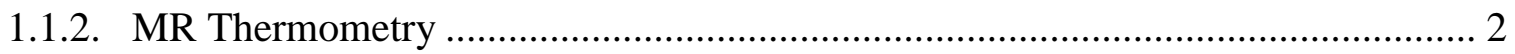

1.1.2.1. Basic Principles behind MR Signal .......................................................... 2

1.1.2.2. Measuring temperature and the Proton resonance frequency shift........... 4

1.1.2.3. Thermometry Artifacts ............................................................................ 6

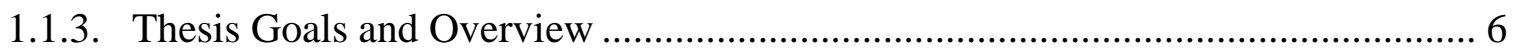

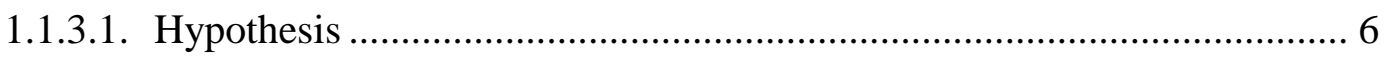

1.1.3.2. Thesis Overview and Organization ..................................................... 7

1.2. Review of MR Thermometry Correction Techniques ....................................................... 7

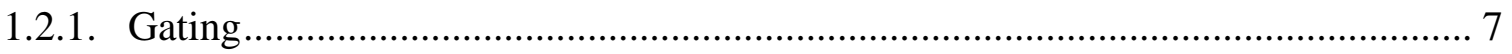

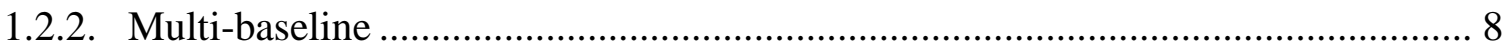

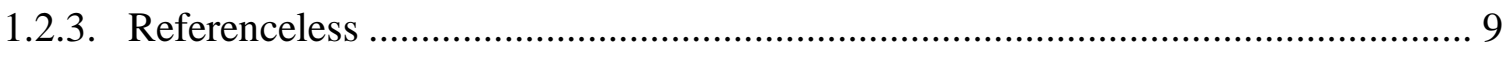

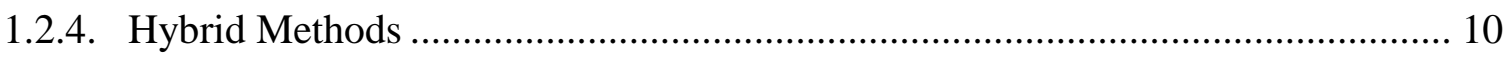

Chapter 2 Principal Component Analysis: Correction of Periodic Artifacts ................................ 11

2.1. Theory behind using PCA as a Multi-baseline Method..................................................... 11

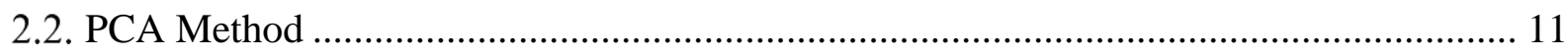


2.3. Experiment Method and Specifications ....................................................................... 13

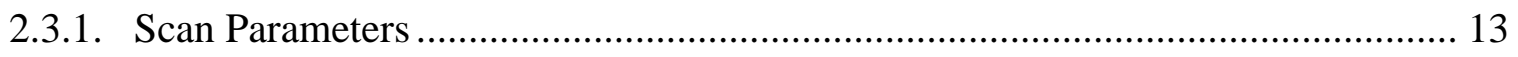

2.3.2. Data Processing Methods................................................................................. 14

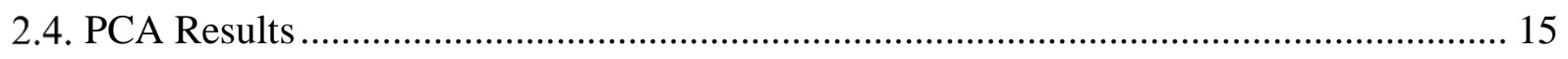

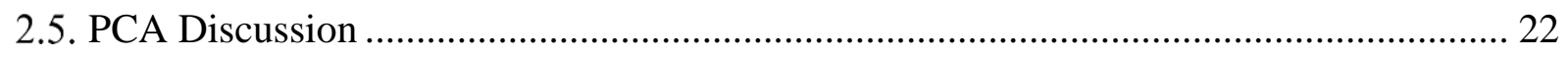

Chapter 3 Projection onto Dipole Fields: Correction of Aperiodic Susceptibility Artifacts ........ 28

3.1. Theory behind using PDF as a Near-Referenceless Method ........................................... 28

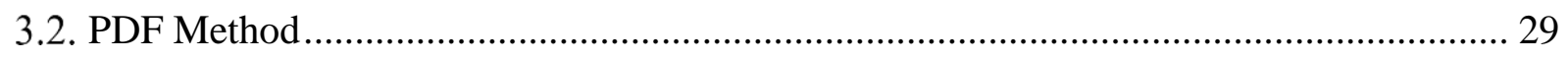

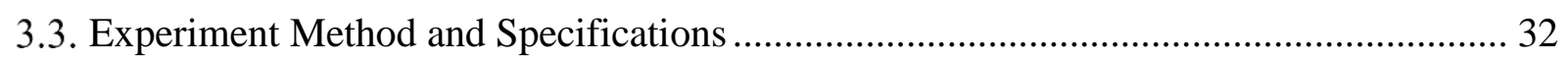

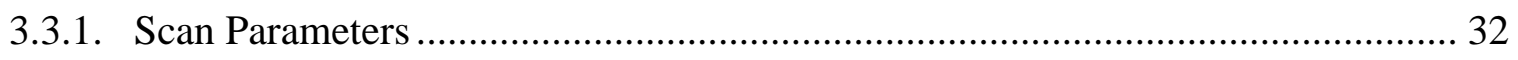

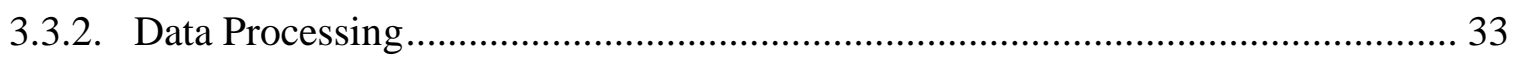

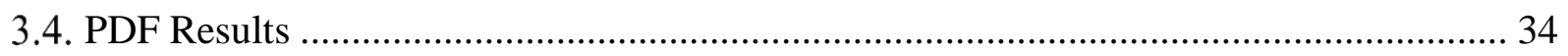

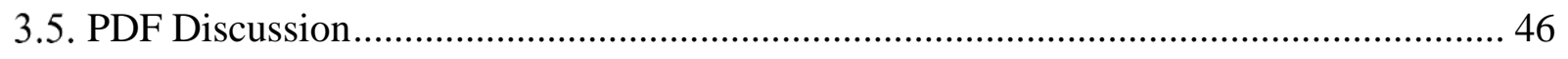

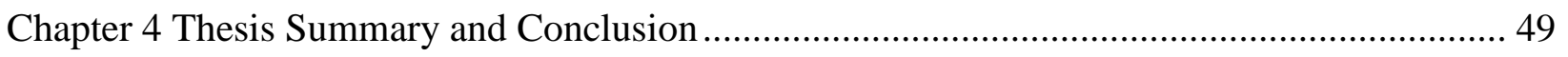

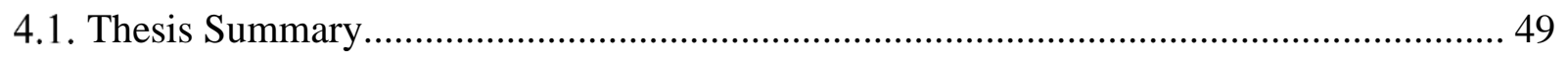

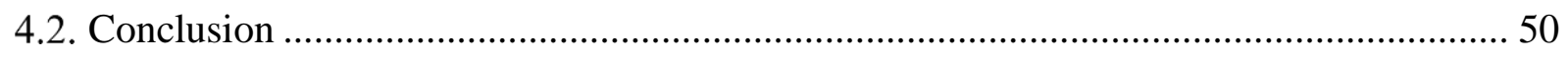

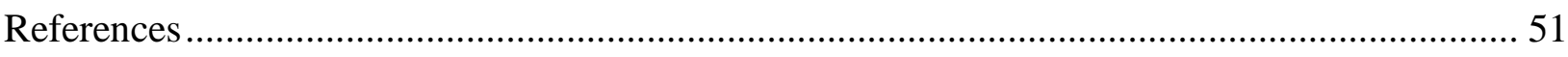

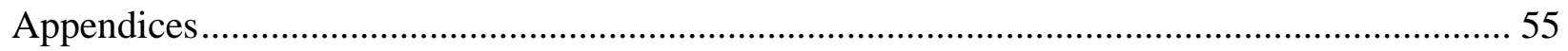

Appendix A. Additional Standard Deviation Maps from PCA Results ........................ 55 


\section{List of Figures}

Figure 1: Cumulative variance accounted for by subsequent eigenvectors .

Figure 2: Porcine kidney: phase image (left), magnitude image (center), and kidney region (right). 16

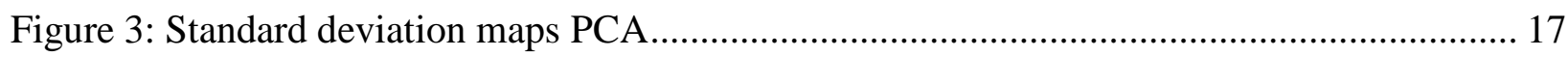

Figure 4: Temperature standard deviation vs. computation time ....................................... 18

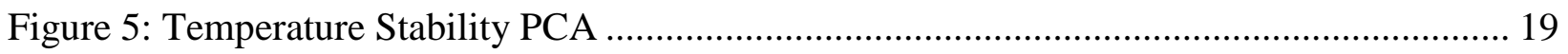

Figure 6: Temperature Precision PCA ............................................................................ 20

Figure 7: Hyperthermia thermometry via standard subtraction and navigator echo LUT.......... 21

Figure 8: Hyperthermia thermometry via standard subtraction and PCA projection ................. 22

Figure 9: Periodic artifacts with a long (blue) and short (green) periods from two different

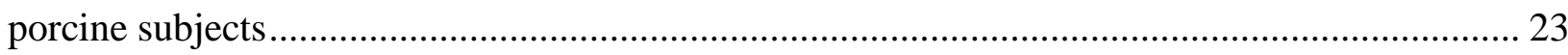

Figure 10: (Top) magnitude image for reference, (bottom) temperature maps with severe artifacts despite low temperature stability and precision.............................................................. 25

Figure 11: Left kidney temperature standard deviation maps, and magnitude image (bottom right)

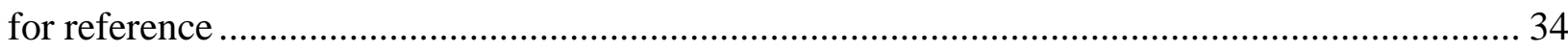

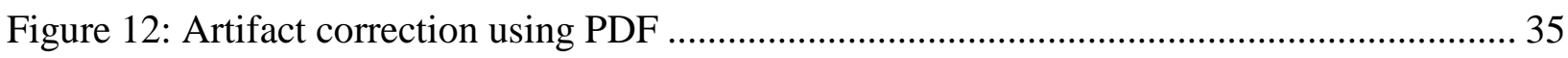

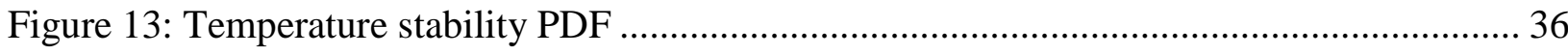

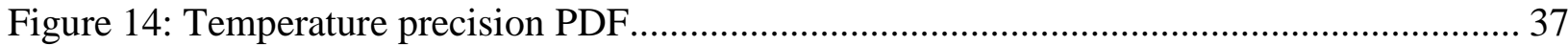

Figure 15: Right kidney temperature standard deviation maps and magnitude image (bottom

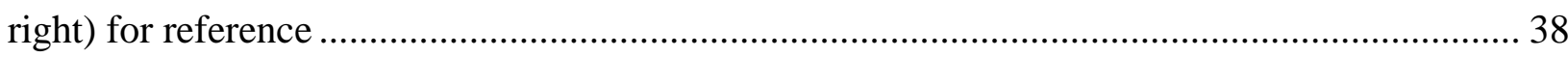

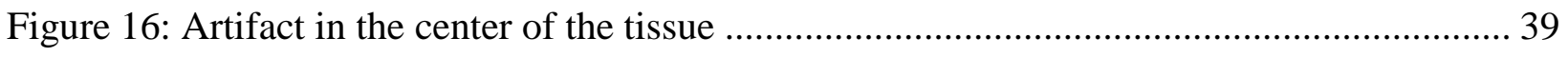


Figure 17: Drift correction using PDF

Figure 18: Human volunteers - temperature stability.................................................... 41

Figure 19: Human volunteers - temperature precision ................................................... 41

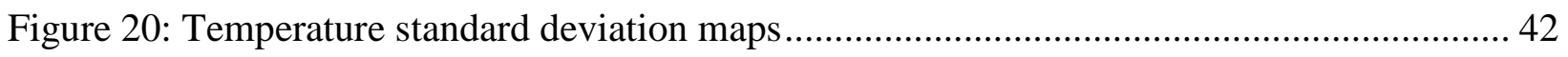

Figure 21: Spatial plot of artifact and polynomial fit correction .......................................... 43

Figure 22: Spatial plot of artifact and PCA-PDF correction............................................... 43

Figure 23: Injected hotspot and PCA-PDF correction ..................................................... 44

Figure 24: Injected hotspot with PCA-PDF error evaluation ............................................... 45

Figure 25: Average theoretical temperature uncertainty ................................................ 45

Figure 26: Progressive correction of artifacts with PCA and subsequently PDF ..................... 46

Figure 27: Standard deviation maps for A.1 right kidney motion dataset .............................. 55

Figure 28: Standard deviation maps for A.2 left kidney motion dataset ................................ 56

Figure 29: Standard deviation maps for A.2 right kidney motion dataset .............................. 57

Figure 30: Standard deviation maps for A.3 left kidney motion dataset ................................ 58

Figure 31: Standard deviation maps for A.3 right kidney motion dataset .............................. 59 


\section{List of Abbreviations}

FOV - Field of view

HIFU - High-intensity focused ultrasound

LUT - Look up table

MR - Magnetic resonance

PCA - Principal component analysis

PDF - Projection onto dipole fields

ROI - Region of interest

SNR - Signal-to-noise ratio

Std. Dev. - Standard deviation

SVD - Singular value decomposition 


\section{Chapter 1 \\ Magnetic Resonance Thermometry for Monitoring Abdominal High-Intensity Focused Ultrasound Procedures}

\subsection{Introduction:}

High-intensity focused ultrasound (HIFU) is a treatment tool with unique qualities. It delivers non-invasive, non-irradiating, local thermal damage. However, unlike radiation, HIFU thermal dose can be hard to predict. Radiotherapy dosage has been extensively modeled and most modern systems calculate precise treatment plans using calibrations to account for the effects of tissue inhomogeneity on the radiation beam [1]. In comparison, HIFU modeling is a more recent endeavor and faces challenges surrounding the mechanisms of HIFU treatment. Specifically, sound wave transmission through various media and structures inside the body can greatly impact the trajectory of sound waves, and organ perfusion and heat diffusion complicate the calculation of heat accumulation [2].

Magnetic resonance (MR) thermometry provides non-invasive temperature measurements within a large field of view. MR thermometry gives crucial image-guidance for HIFU procedures. Unfortunately, motion and magnetic susceptibility changes create severe MR thermometry artifacts [3].

\subsubsection{Abdominal HIFU}

HIFU's unique advantages make it well suited for creating localized thermal lesions in diseased tissue that is difficult to access; involves high risk of blood loss or nerve damage; or for cases which are completely inoperable. Clinical needs are diverse, including cancer in the kidneys, liver, and pancreas, adrenal glands, spleen, and colorectal tissue.

Of particular interest are the renal and adrenal gland regions which are common sites for neuroblastoma. In 2015, neuroblastoma and other peripheral nervous cell tumours accounted for $8 \%$ of cases and $11 \%$ of cancer deaths for children 0-14 years of age in Canada [4]. In an effort to improve outcomes, high-risk patients are often given aggressive multimodal treatments including intensive chemotherapy, radiation therapy, surgery, stem cell transplantation, and 
immunotherapy [5]. The combination of these therapies can take a great toll on pediatric patients and necessitates extensive multi-disciplinary follow-up [5].

HIFU offers the opportunity to replace or locally enhance these standard therapies while reducing patient harm. The smaller anatomy of pediatric patients can also make traditional surgery more challenging. As an alternative, HIFU may help reduce the risks associated with invasive surgery. Pediatric patients can also be good candidates for HIFU because their smaller anatomy may allow soundwaves to reach deeper targets more easily. At the same time, smaller targets require accurate HIFU monitoring to ensure the safety of other critical structures in close proximity.

\subsubsection{MR Thermometry}

MR phase is sensitive to temperature changes via the proton resonance frequency shift. This allows for rapid temperature calculation and measurement. Calculating the relative temperature change between two dynamic images only requires the subtraction of the phase images and the conversion from phase change to temperature change via the following equation (1.1).

$$
\Delta T=\frac{\phi(T)-\phi\left(T_{o}\right)}{\gamma \alpha B_{o} T E}(1.1)[6]
$$

Where $\Delta T$ is the change in temperature, and $\phi(T)$ is the phase at the current temperature, $T$, or reference temperature, $T_{o}$. In the denominator, $\gamma$ is the gyromagnetic ratio, $\alpha$ is the proton resonance frequency change coefficient, $B_{o}$ is the magnetic field strength, and $T E$ is the echo time.

This calculation is simple and only requires acquisition of two images. As such, MR thermometry is ideal for high temporal resolution monitoring of thermal treatments. Unfortunately, many clinically relevant areas exhibit severe thermometry artifacts.

\subsubsection{Basic Principles behind MR Signal}

In magnetic resonance imaging, nuclear magnetic moments of the hydrogen atoms are probed to investigate the sample. Individual nuclear magnetic moments are caused by proton spin and are proportional to the angular momentum of the proton spin. Hydrogen proton spins have two energy eigenstates, spin-up and spin-down [7]. Individual spins exist as a weighted combination 
of these two states. Using complex coefficients, these eigenstates form a vector basis that spans three-dimensional space. More intuitively, these two states can be considered analogous to azimuthal and polar angles, which can produce a unit vector in any direction in threedimensional space.

In a strong magnetic field, there is a slight tendency for magnetic moments to point in the same direction as the field. Quantitatively, the magnitude of this tendency can be calculated using Boltzmann's distribution

$$
N_{\text {aligned }} / N=\exp \left[\frac{-\Delta E}{k T}\right](1.2)[8]
$$

Where $N_{\text {aligned }} / N$ is the ratio of aligned, to non-aligned spins, $\Delta E$ is the energy gap between the two states, $k$ is Boltzmann's constant, and $T$ is the temperature. This equation (1.2) accounts for environmental and characteristic properties of the system. The slight surplus of spins in the lower energy state represents the portion of spins contributing to macroscopic net magnetization aligned with the magnetic field.

All spins within the field also exhibit a precession phenomenon about the field direction. The main magnetic field exerts force on nuclear magnetic moments, turning them to the orientation of the field. However, the angular momentum of spins resists this change in orientation, resulting in precession [8]. The rate of precession is proportional to the gyromagnetic ratio and the strength of the applied magnetic field. The gyromagnetic ratio is the ratio of the angular momentum to the magnetic moment of a spin. Note that while the precession rate is independent of the angle of precession, the precession between spins is not naturally in-phase, thus off-axis components cancel. The resulting net magnetization is thus aligned with the main magnetic field.

Although individual spins precess, there is no macroscopically measurable oscillating response. To elicit a measurable signal, this net magnetization must be perturbed using an oscillating magnetic field, usually delivered as a pulse. Energy transfer to spins is most efficient at resonant (precession) frequency. As such, an oscillating homogenous $B_{1}$ field tips the entire spin population by a flip angle determined by the duration and magnitude of the pulse [7]. This rotates the entire spin population and causes the net magnetization to tip off of the axis of the field. The small percentage of spins, aligned parallel to the magnetic field, is tipped 
simultaneously from the longitudinal axis (parallel to the main magnetic field) toward the transverse plane (perpendicular to the field). Shifting together, these spins are in-phase and take a spiral path toward the transverse plane. Being in-phase, the precession of these spins, or equivalently the precession of the net magnetization, produces a measurable changing magnetic field. This changing magnetic field is converted to electrical voltage signals via MR coils.

Over time, spins lose their energy through interactions with their environment. This relaxation, called T1 longitudinal relaxation, eventually restores spins to their equilibrium, recovering a net magnetization in the direction of the main magnetic field [9]. Concurrently, the measurable signal is also decaying due to dephasing of the spins that began their transverse precession inphase. This relaxation (T2 transverse relaxation) can be caused by i) magnetic field inhomogeneities at the macroscopic or molecular scale, or ii) inter-spin energy exchanges [9]. The latter (ii) can have a dephasing effect while causing a net zero change in longitudinal relaxation. These relaxation phenomena depend on the characteristics of the spins and their environment. Spins in different tissues thus relax at different rates, giving rise to image contrast.

\subsubsection{Measuring temperature and the Proton resonance frequency shift}

In MR thermometry, there are several methods to measure temperature change. Each method exploits a different MR parameter's sensitivity to change in temperature. These include proton density, $T_{1}$ and $T_{2}$ relaxation times, diffusion coefficient, magnetization transfer, and proton resonance frequency [6]. Unfortunately, many of these methods, including proton density, $T_{1}$ relaxation, and diffusion, require long acquisition times which limits the temporal resolution of the thermal imaging. Additionally, proton density, $T_{2}$ relaxation, and magnetization transfer methods have low temperature sensitivity and thus need high SNR imaging. These measurements can be further complicated by the fact that some temperature sensitivities are tissue dependent ( $T_{1}$ and magnetization transfer), or non-linear $\left(T_{2}\right)$. The phase subtraction method using change in proton resonance frequency is the most viable metric because i) its temperature relationship is linear over a wide range of temperatures, ii) it is largely tissueindependent (for aqueous tissues), and iii) it can be measured with very high temporal resolution [6]. Note that there is also a way to use the proton resonance frequency as a spectroscopic method to measure absolute temperature by using adipose tissue as a temperature insensitive reference. However this method has a more limited temporal and spatial resolution [6]. As such, 
the phase subtraction method using the proton resonance frequency is the most suitable and is used throughout the remainder of this work.

Measuring change in temperature with the proton resonance frequency method is achieved via subtraction across dynamic phase images. The phase of a collection of spins represents the amount of precession that has taken place from the time of the $B_{1}$ pulse to the time the signal is measured. In a completely homogenous sample, with a perfectly uniform field, the phase should be equal at all points in the image. Relative phase differences occur predominantly due to field inhomogeneity. The most important sources to consider are

o inhomogeneity of the main magnetic field,

- magnetic susceptibility of the sample, and

- chemical shift of hydrogen protons in water

The chemical shift factor is temperature dependent and is used in the temperature calculation. This chemical shift factor refers to the notion that the resonance frequency of a given proton is influenced by its local chemical environment, i.e. molecular structure. In the case of water, hydrogen bonds impact the level of electron shielding experienced by the hydrogen proton. The equation below (1.3) displays the proton resonance frequency with dependence on shielding.

$$
\omega=\gamma B_{o}(1-s)(1.3)[6]
$$

Where $\omega$ is the resonance frequency, $\gamma$ is the gyromagnetic ratio, $B_{o}$ is the magnetic field strength, and $s$ is the shielding constant which is temperature dependent.

Hydrogen bonds in water undergo rupture, stretching, or bending when heated, leading to an average increase in electron shielding of protons [6], [10]. This shielding reduces the effective magnetic field experienced by the hydrogen protons and thus decreases their precessional frequency. A heated region experiencing this chemical shift will accumulate phase difference. Calculating the relative temperature change requires a reference phase image that contains only phase differences related to this chemical shift. Phase effects due to field inhomogeneity and magnetic susceptibility must be identical in both phase images. When these two factors are not identical in both phase images, they create artifacts in the temperature calculation. 


\subsubsection{Thermometry Artifacts}

Thermometry artifacts occur when there are phase differences caused by factors other than temperature change. Magnetic susceptibility differences between tissues and most prominently between air and tissue create field inhomogeneity. With different regions experiencing different strengths of effective magnetic field, precessional frequencies shift leading to dephasing. This gives tissue an inherent phase pattern related to its geometry. Tissue motion causes these patterns to become misaligned in consecutive dynamics, leading to thermometry artifacts.

Changes in air volume surrounding tissue can also modify the inhomogeneity and change the inherent phase pattern. This change in background susceptibility can occur due to motion of the tissue relative to the surrounding air. Alternatively, changes in air volume can occur without any visible motion in the magnitude images. For example air in the lungs or bowel can cause artifacts even when they are outside of the FOV.

\subsubsection{Thesis Goals and Overview}

\subsubsection{Hypothesis}

The goal of this work is to develop an algorithm for artifact correction in MR thermometry. Also, in order to possess any real utility, the algorithm must operate within the realm of real-time processing. As such, this thesis aims to test the hypothesis that:

Principal component analysis (PCA) and projection onto dipole fields (PDF) can be used to correct MR thermometry. Specifically,

A) to an accuracy of $\pm 1{ }^{\circ} \mathrm{C}$ within the targeted cell and to $\pm 3{ }^{\circ} \mathrm{C}$ in the periphery, and

B) having a processing time, for a single dynamic, less than the dynamic scan time of the MR sequence.

These metrics are based on the limit of accuracy of artifact-free MR thermometry in soft tissue. This was found to be about $0.91{ }^{\circ} \mathrm{C}$ in a patient study using similar scan parameters to the ones used in this work [11]. Additionally, certain applications employ chemotherapy in conjunction with HIFU using thermosensitive-liposomes. These are typically activated by inducing mild hyperthermia at $41^{\circ} \mathrm{C}$, which is just below the limit of $43^{\circ} \mathrm{C}$ at which necrosis can begin in the 
surrounding healthy tissue [12]. It is above $43^{\circ} \mathrm{C}$ that cells are no longer able to develop thermal tolerance during heating; this leads to a doubling of the cell death rate for every degree above this breakpoint [13]. As such, accuracy to $\pm 1{ }^{\circ} \mathrm{C}$ represents a challenging, but warranted goal.

The targeted cell in A) refers to a treatment cell, typically with a 4-mm radius, specified by the user in the HIFU treatment planning tool. Also, note that part B) does not account for computational overhead such as graphics and disk storage; instead it reflects the minimum requirement for this process to be considered feasible for real-time operation.

\subsubsection{Thesis Overview and Organization}

This thesis presents a hybrid algorithm using two techniques, PCA and PDF. After a review of existing correction strategies (Section 1.2), both PCA and PDF are described in detail. Each chapter covers the theory behind the method, the method details, experiment specifications, results, and discussion. The last chapter offers a brief summary and potential future directions.

\subsection{Review of MR Thermometry Correction Techniques}

Artifacts occur when phase changes for reasons other than temperature, i.e. motion or susceptibility changes. To have artifact-free thermometry, the reference phase image must match the dynamic phase image. This match can be achieved by manipulating either the dynamic phase image or the reference phase image.

\subsubsection{Gating}

Gating is a simple but effective method of controlling the dynamic phase image. Using navigator echoes [14], or extracorporeal sensors such as respiratory belts [3], cameras [15], or ultrasound imaging probes [16], the MR sequence can be triggered to acquire images only when the tissue is in a particular position. This can be further controlled by regulating breathing with a ventilator. There are several issues with these methods including i) under-defined tracking, ii) limited sonication duty cycle, and iii) ventilator contraindications.

i) The typical tracking tools use a single 1D measurement to track the position of the target undergoing 3D non-rigid motion. This can lead to under-defined tracking which fails to determine when the tissue is in the correct position. 
ii) Certain treatments such as hyperthermia, require continuous, low-power heating, which is hard to achieve if sonications can only be delivered once every respiratory cycle.

iii) Using a ventilator, sonication duty cycle can be improved by forcing prolonged periods of apnea or by inducing hyperventilation to limit the range of motion. However, these are not always possible and depend on the patient's condition.

\subsubsection{Multi-baseline}

As opposed to gating, which waits for the tissue position to match the reference image, it is possible to prepare a reference image for many tissue positions. This is the premise of the multibaseline strategies [17], [18]. Often these methods still rely on similar tracking tools to gating methods in order to match captured images with the closest reference image available. Navigator echoes are the method of choice for several techniques [17], [19]. As such, many of the same problems found in gating techniques also apply.

The method proposed by [18] replaces these tracking tools with the use of the correlation coefficient between images. The atlas image that gives the highest correlation coefficient when compared with the new dynamic is selected as the closest match. Image registration is then used to calculate a motion vector field to determine the motion from i) the original tissue position to ii) the position found in the reference image. This motion vector field is then applied to shift the phase of the new dynamic image into the original tissue position (i). Unfortunately, correlation coefficients can be highly sensitive to small differences and even noise, which could potentially lead to inaccurate reference selection. Additionally, both the correlation and the image registration processes only consider changes in the magnitude images, which do not linearly correspond to changes in phase images when there are susceptibility artifacts. Even advancements on this method using PCA [20], [21], only improve stability of the image registration by reducing noise contributions to the motion vector fields. This still does not account for any susceptibility effects that are not expressed in the magnitude images.

Perhaps more comprehensive is the simple projection method in [22]. In this method, a new dynamic complex image is projected onto the subspace spanned by all complex images in the atlas. This orthogonal projection gives the linear combination of atlas images which best matches 
the new dynamic. This method is simple and effective, but may not be the most efficient representation of the atlas data.

Furthermore, all multi-baseline methods are inherently limited to correcting phase changes recorded in the atlas. This inability to compensate for aperiodic artifacts is one of the major motivations for referenceless methods.

\subsubsection{Referenceless}

Referenceless methods, in theory, offer a solution to the shortcomings of both gating and multibaseline strategies. The premise is to use a model to approximate the phase in absence of heat [23]. This model must be able to recreate all plausible non-heated phase patterns. Smooth, homogenous phase patterns are easy to model with low-order polynomials. But this does not account for all phase patterns found in tissue. Basic forms of this method use the phase information in a frame around the ROI to interpolate the phase within the ROI using low-order polynomials [23]. This strategy relies on user input to specify the ROI and frame. This selection is critical because any heating in the frame will alter the results of the interpolation.

The near-harmonic model offers more physically meaningful phase interpolation [24]. It exploits the fact that in regions of homogenous or linearly-changing susceptibility, the phase map will be a harmonic function [24]. This improves the stability of the solution and is designed to avoid incidents of near-singular matrix inversion that lead to errors in the polynomial fit methods. It only requires a very thin border of phase information to recover the phase within the ROI. This circular border can even be discontinuous, as long as i) individual gaps do not exceed a $\frac{\pi}{2}$ radian angle, and ii) phase data is provided for at least $60 \%$ of the perimeter [24].

While this near-harmonic method is more robust, its stringent requirements limit applicability. Regions must have homogeneous or linearly-changing susceptibility and the border must be well defined in a non-heated, artifact-free region. This implies that the border must be small enough to exclude regions of inhomogeneous phase, yet large enough to avoid all heat diffusion from the target. It also constrains temperature mapping to a small FOV around the target, potentially failing to report unintended heating in peripheral regions. Perhaps most restrictive is the fact that this border must be carefully defined for any given tissue position. The published data on this method used a gated acquisition sequence to ensure consistent conditions. Developing an 
implementation for real-time dynamic motion is substantially more challenging. The authors suggested automated segmentation strategies [24], however, the entire method's accuracy is heavily dependent on this border selection. This means the artifacts must be accurately identified and avoided before correction can occur. Sadly, this points to the fact that many referenceless methods are only suitable when the phase is already very stable and highly predictable.

Arguably the reweighted strategy [25] offers the most flexible solution because it can progressively mask out areas of high spatial frequency (i.e. hotspots), without any user interaction. In this method, a low-order polynomial function is used to model the phase and regions that achieve a poor fit are given progressively less contribution to this fitting. In this way the hot-spots which do not fit the low-order polynomial will be automatically masked out. Still, this adaptive high-pass filtering has no way of meaningfully discriminating between high spatial frequency artifacts and hotspots.

\subsubsection{Hybrid Methods}

Combining multi-baseline and referenceless approaches helps to form a more comprehensive solution. Multi-baseline methods can easily correct for inhomogeneous phase patterns with high spatial frequency features as long as these patterns occur periodically. Meanwhile referenceless methods can help with aperiodic artifacts that have a wide-spread effect, but are unable to mimic complex phase patterns on their own. The various multi-baseline and referenceless methods can be combined by either alternating between the two or using both in concert. For example, one hybrid method, [26], uses a multi-baseline method as the default correction and switches to a referenceless approach when the measured motion exceeds the range recorded in the atlas. In contrast, another method uses the projection-based multi-baseline technique and then feeds any residual error to a reweighted polynomial fit scheme [22]. For the purpose of comparison, this hybrid projection and reweighted polynomial fit method will be considered as the state of the art

benchmark. This method was recreated for comparison using a projection-based atlas and a $6^{\text {th }}$ order polynomial fit scheme. 


\section{Chapter 2 \\ Principal Component Analysis: Correction of Periodic Artifacts \\ 2.1. Theory behind using PCA as a Multi-baseline Method}

In theory, the atlas of a multi-baseline method needs an infinite number of reference images to map the continuous motion of the tissue. However, this is neither feasible nor necessary and good approximations can generally be made using very few images. One of the earliest multibaseline systems was proposed using 50 reference images [18]. In order to determine the closest matching reference image, many approaches rely on motion tracking tools. However, none of these tools can accurately track phase changes within the entire 2D FOV. Furthermore, tracking continuous motion offers little advantage because the reference image ultimately comes from a finite atlas of discrete snapshots. From an informatics perspective, matching a newly acquired image with the correct reference in the atlas can be considered as an image recognition problem. As such, the proposed PCA method was adapted from previous work in the field of facial recognition [27]. Their design provides a robust and efficient method to find the closest reference in the atlas.

To reduce the discretization error that inherently comes from working with a finite number of reference images, some methods also use linear interpolation between reference images [28],[19]. This helps to artificially increase the sampling density of the respiratory cycle. In this case, PCA would help reduce redundant storage and computation by solving for an efficient basis that spans the variation. In this way PCA can exploit large amounts of atlas data while minimizing storage and computation costs.

\subsection{PCA Method}

This PCA method is adapted from work in facial recognition [27]. First an atlas of images is accumulated before any heat is applied. This forms a $n \times p$ matrix, where $n$ is the number of images in the atlas and $p$ is the total number of pixels in a single image. Once complete, singular value decomposition (SVD) is used to decompose the atlas into principal vectors, referred to as eigenimages in the factorization below (2.1). 


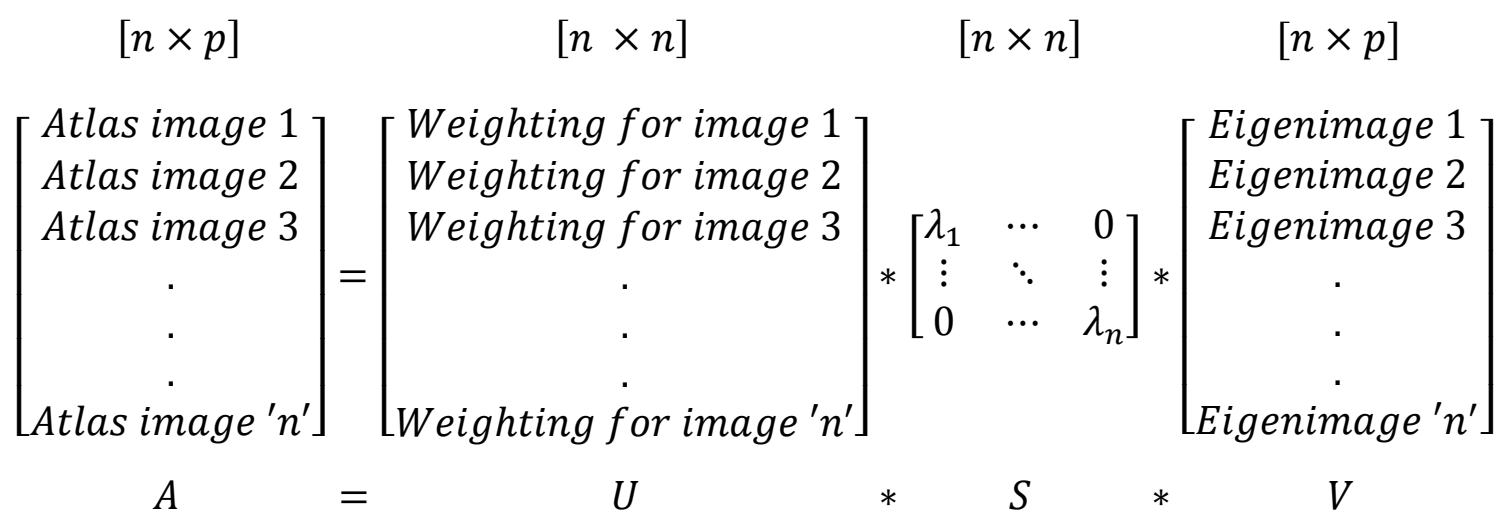

These principal vectors form a new basis that span the same $p$-dimensional subspace as the original atlas images. The advantage is that the principal vectors are a more efficient basis, i.e. the majority of the information is stored in the lower rank vectors. As such, it is possible to achieve a very close approximation using only a fraction of the $n$ vectors.

The reference image can then be calculated as a weighted sum of the $m$ principal vectors, where $m<n$, by finding the least-squares solution to (2.2).

$$
[1 \times m] \quad[m \times p] \quad[1 \times p]
$$

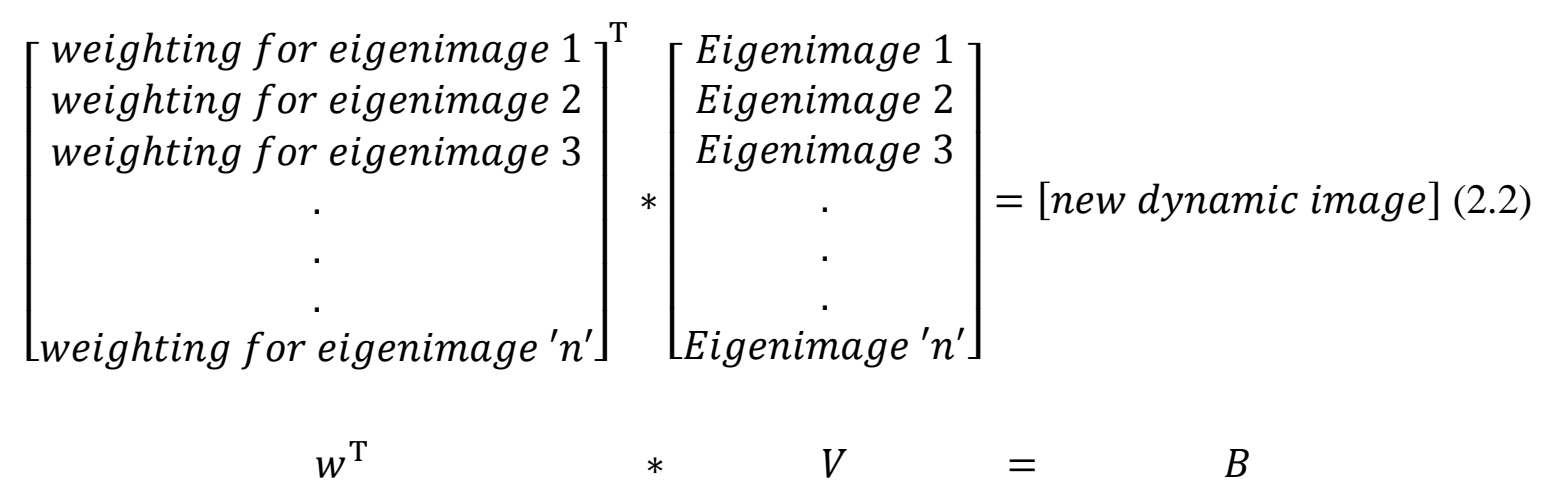

Note that $m$ is user selected, but this can easily be automated so that the $m$ principal vectors account for a user-specified percentage of the variance. Determining the optimal $m$ will depend on the computation time restraints of the protocol at hand. In this work $m$ is selected to account for at least $99.9 \%$ of the variance of the first 50 images. This preserves the majority of the useful information while demonstrating PCA's removal of redundant data. Note that the proportion of the total variance, that each principal vector accounts for, can be found by calculating the 
eigenvalues of each principal vector. The eigenvalues are the square of the singular values, $\lambda_{i}$ where $1 \leq i \leq n$, found in $S$, in (2.1).

Once $w^{\mathrm{T}}$ is determined, the reference image $B_{r e f}$ can be used to remove periodic artifacts

$$
\begin{gathered}
w^{\mathrm{T}} * V=B_{\text {ref }}(2.3) \\
\Delta \phi_{P C A}=B-B_{\text {ref }}(2.4)
\end{gathered}
$$

Here $\Delta \phi_{P C A}$ represents the phase difference calculated using the PCA reference.

\subsection{Experiment Method and Specifications}

\subsubsection{Scan Parameters}

A) Kidney motion datasets:

A series of in vivo experiments were performed to test the motion correction algorithms in porcine kidneys. The porcine subjects were anesthetized and ventilated at a rate of approximately 22 breaths/min. Note there was no hyperventilation, apnea control, or intentional control of the respiratory rate for the purpose of artifact reduction. Three subjects were scanned on both sides, totaling 6 kidney scans. Imaging was performed on Philips 3T Achieva scanner using a T1weighted gradient recalled echo (GRE), echo planar imaging (EPI) sequence with the following parameters: FOV: $256 \times 256 \mathrm{~mm}^{2}$, voxel size $=1.5 \mathrm{~mm}$, slice thickness $=7 \mathrm{~mm}$, TE/TR $=$ $19.5 / 30 \mathrm{~ms}$, flip angle $=19.5^{\circ}$, acquisition matrix $=172 \times 171$, reconstruction matrix $=176$, ETL $=9, \mathrm{NEX}=1$, slices $=6$, dynamic time $=3.44 \mathrm{~s}$. A 32 channel cardiac receive coil was used in this experiment.

\section{B) Sonication datasets}

Data from in vivo porcine head and neck hyperthermia experiments [19] were also processed. In this study, the neck region was sonicated for 30 minutes under manual control with a target temperature of $41{ }^{\circ} \mathrm{C}$ [19]. This study also used a GRE-EPI sequence on a Philips 3T Achieva scanner, but with following parameters: FOV: $400 \times 400 \mathrm{~mm}$, voxel size $=2.08 \mathrm{~mm}$, slice thickness $=7 \mathrm{~mm}, \mathrm{TE} / \mathrm{TR}=16 / 45 \mathrm{~ms}$, flip angle $=18^{\circ}$, acquisition matrix $=192 \times 191$, 
reconstruction matrix $=192, \mathrm{ETL}=11, \mathrm{NEX}=1$, slices $=4$, dynamic time $=3.07 \mathrm{~s}$. Abdominal surface coils were used in this experiment.

\subsubsection{Data Processing Methods}
A) Kidney motion datasets

The kidney motion datasets were processed in four ways:

1) Standard subtraction,

2) Multi-baseline using projection onto an atlas of 50 images,

3) PCA multi-baseline using projection onto an atlas of $m$ eigenimages derived from 50 images, and

4) Multi-baseline using projection onto an atlas of $m$ images

Here $m$ represents the lowest number of eigenimages that cumulatively account for at least 99.9\% of the variation within the first 50 images. For example, in the dataset below (Figure 1), the first 4 eigenimages account for more than $99.9 \%$ of the total variation within the first 50 dynamics. This is different for each dataset and the $m$ values are reported alongside the standard deviation maps in Figure 3: Standard deviation maps: Table indicates number of dynamics used in the atlas, computation time, and standard deviation in the kidney for each processing method. Magnitude image gives orientation of standard deviation maps. The subtraction method (1) produces substantial artifacts extending into kidney region. The 50 image atlas (2) achieves the best correction and PCA (3) has similar results. The 4 image atlas (4) provides limited correction based on the small number of images in its atlas. and Appendix A. For example, method 3) would only use 4 eigenimages in the projection calculation. 


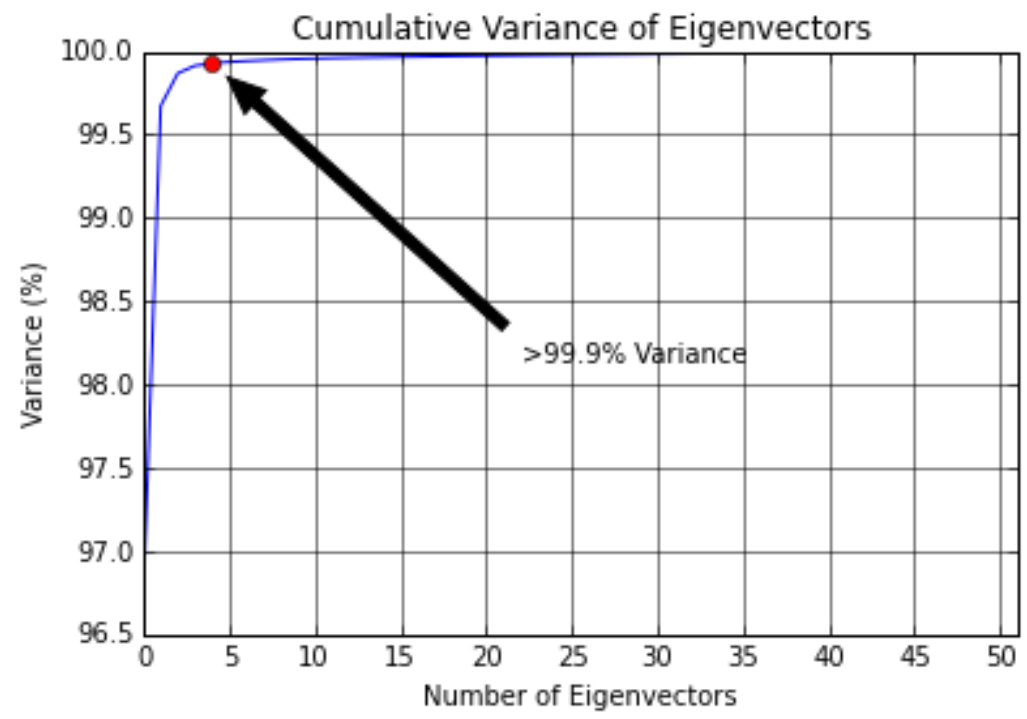

Figure 1: Cumulative variance accounted for by subsequent eigenvectors: The majority of the variance is contained within the first few eigenvectors. Over $99.9 \%$ of the variance is stored within the first 4 eigenimages. The remaining 46 eigenimages are largely redundant and can be discarded to improve computational efficiency.

Method 4) uses projection onto only the first $m$ dynamics. As such, method 3) projects onto $m$ eigenimages derived from the information within the first 50 dynamics, while method 4) projects onto $m$ images with only the information occurring in the first $m$ dynamics. This provides a way to quantify the advantage of using a condensed representation of a longer observation period.

B) Sonication dataset

The sonication dataset was processed using a 16 image atlas without dimensionality reduction. This data is also processed using standard subtraction and using a navigator echo look-up-table (LUT) method [19]. This comparison is used to evaluate the accuracy of projection-based techniques as opposed to methods that use supplementary motion tracking tools.

\subsection{PCA Results}
A) Kidney motion datasets

Below is an example of a magnitude and phase image from one of the datasets (Figure 2). 

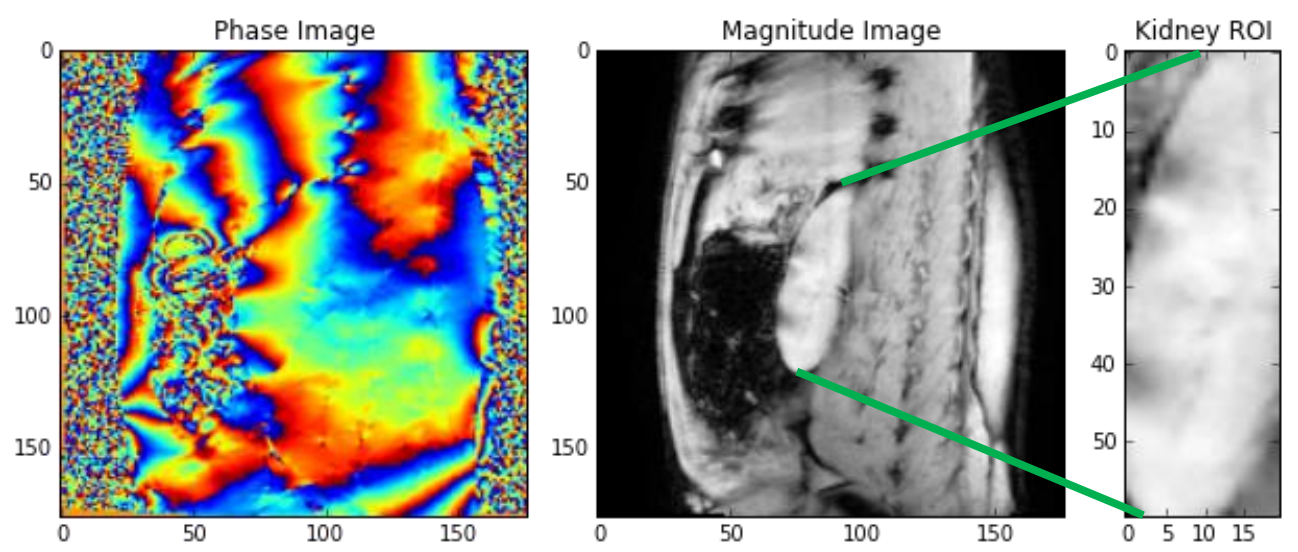

Figure 2: Porcine kidney: phase image (left), magnitude image (center), and kidney region (right). Note that organ boundaries can be clearly delineated in magnitude image, but it is much more difficult to distinguish and separate components in the phase image.

The standard deviation of each dataset is calculated by taking the temporal standard deviation of each pixel's temperature values. Note that before the standard deviation is calculated, the temperature maps are registered using an optical flow algorithm. This prevents the movement of artifacts from artificially raising the variation and helps ensure that the variation reported for a pixel or ROI is only a result of the thermometry in that region. These maps are shown below for one dataset using the four processing methods (Figure 3). The standard deviation maps display the severity of the artifacts remaining after processing via each method. Also shown is average computation time and mean standard deviation within the entire kidney. Note that computation time is the average amount of time taken to generate a single reference image $(0 \mathrm{~ms}$ for the subtraction method). 


\begin{tabular}{|l|r|r|r|r|}
\hline Dataset & \multicolumn{4}{|c|}{ A.1 Left Kidney } \\
\hline Processing Method & 1 & 2 & 3 & 4 \\
\hline Initial Number of Images in Atlas & 0 & 50 & 50 & 4 \\
\hline Final Number of Images in Atlas & 0 & 50 & 4 & 4 \\
\hline Average Computation Time (ms) & 0 & 153 & 18 & 18 \\
\hline Standard Deviation in Kidney $\left({ }^{\circ} \mathrm{C}\right)$ & 1.98 & 0.41 & 0.52 & 1.17 \\
\hline
\end{tabular}
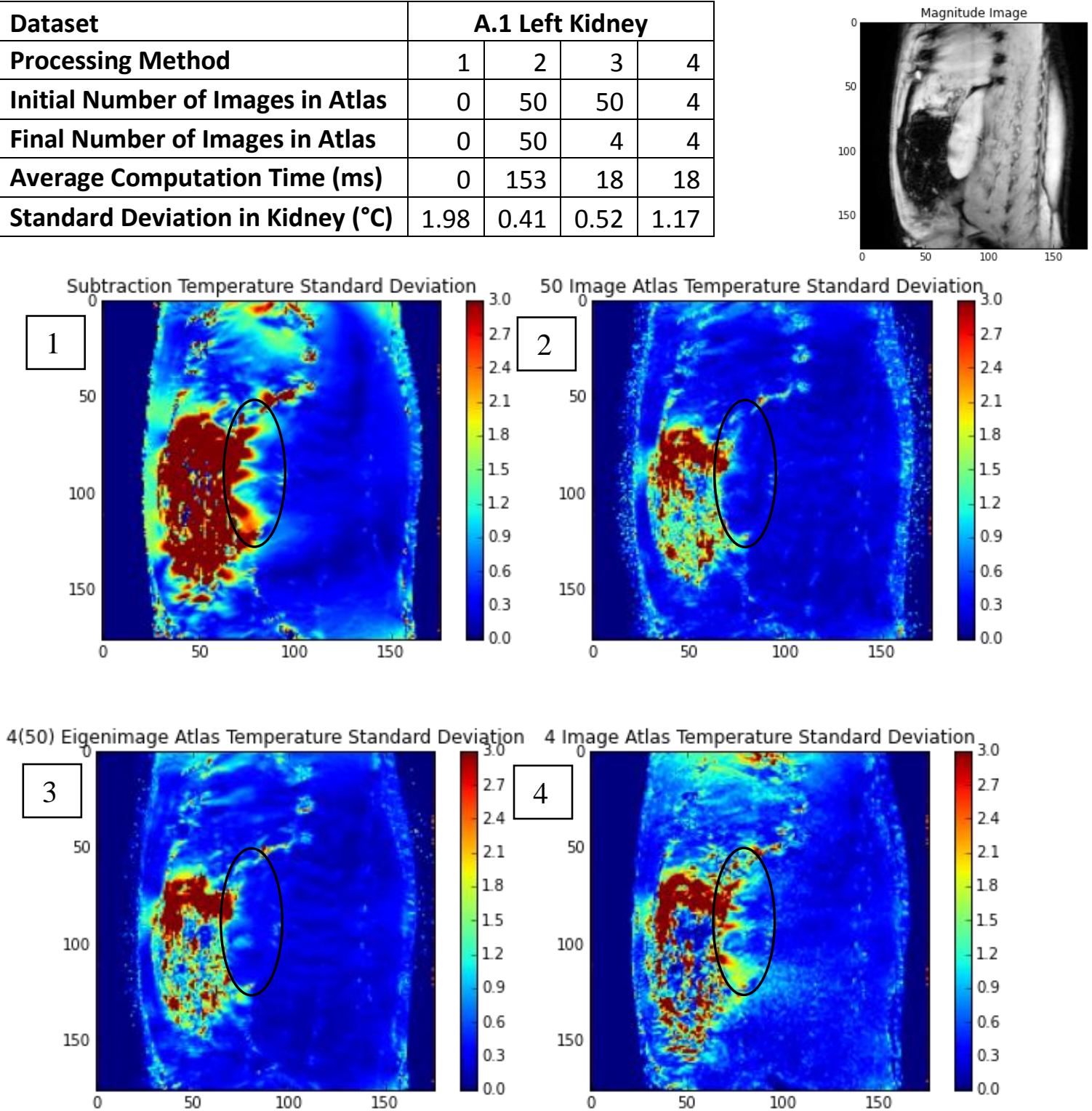

Figure 3: Standard deviation maps: Table indicates number of dynamics used in the atlas, computation time, and standard deviation in the kidney for each processing method. Magnitude image gives orientation of standard deviation maps. The subtraction method (1) produces substantial artifacts extending into kidney region. The 50 image atlas (2) achieves the best correction and PCA (3) has similar results. The 4 image atlas (4) provides limited correction based on the small number of images in its atlas.

These standard deviation maps indicate that the PCA correction (map 3, std. dev. $=0.52{ }^{\circ} \mathrm{C}$ ) is comparable with the 50 image atlas method (map 2, std. dev. $=0.41{ }^{\circ} \mathrm{C}$ ). The advantage is that the computation time is equivalent to that of the 4 image atlas method, which exhibits higher 
standard deviation due to its limited observation period (map 4, std. dev. $=1.17{ }^{\circ} \mathrm{C}$ ). Additional standard deviation maps for the other datasets can be found in Appendix A.

Figure 4 graphs the standard deviation for all datasets, within a smaller ROI in the kidney, against the average computation time required to generate a reference image.

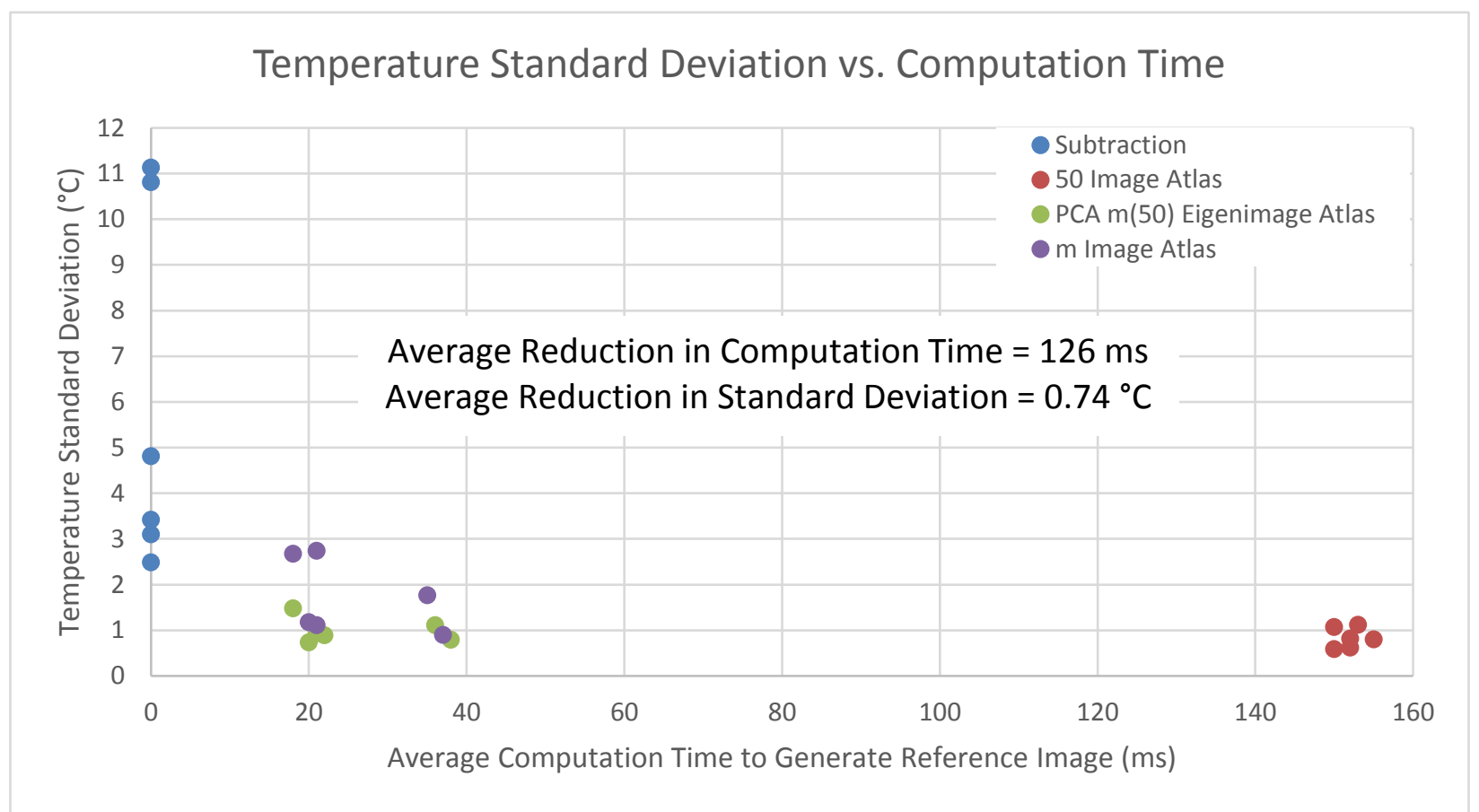

Figure 4: Temperature standard deviation vs. computation time: PCA (green) achieves the best compromise between standard deviation and computation time. On average PCA is $126 \mathrm{~ms}$ faster than the 50 image atlas (red) and reduces standard deviation from $1.73{ }^{\circ} \mathrm{C}(\mathrm{m}$ image atlas method, purple) to $0.99{ }^{\circ} \mathrm{C}$ (PCA, green), a $43 \%$ improvement.

The 50 image atlas (method 2) offers the lowest standard deviation, but has the highest computational demands. The PCA method reduces the average computation time by $126 \mathrm{~ms}$ across all datasets, as compared with method 2. The $m$ image atlas (method 4 ) provides reasonably good correction, however PCA reduces the average standard deviation by about 0.74 ${ }^{\circ} \mathrm{C}$ with no additional computational costs.

A more thorough assessment of the correction accuracy can be seen in the temperature stability and precision graphs below (Figure 5 and Figure 6). Temperature stability is calculated as the temporal standard deviation of the spatial average of the temperature within a ROI. 


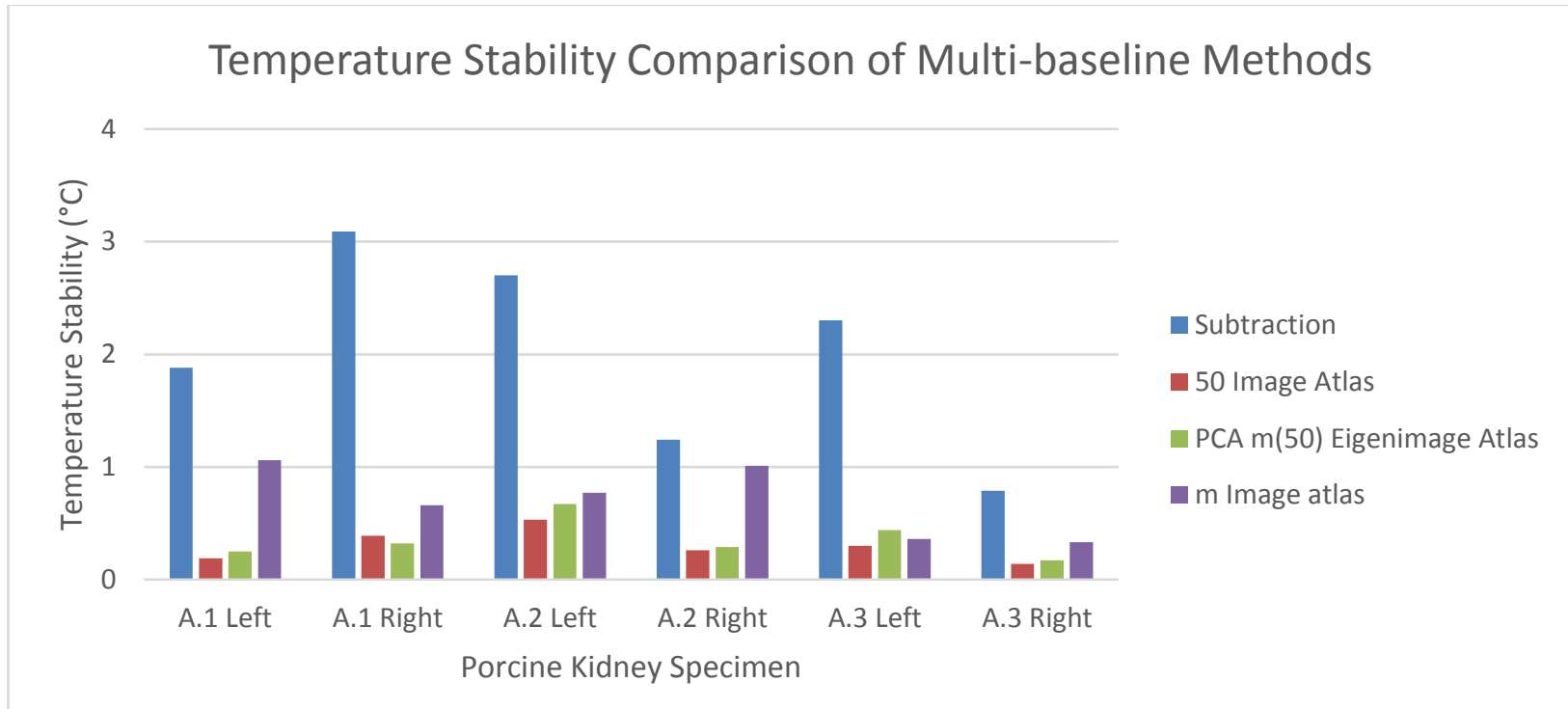

\begin{tabular}{|l|c|c|c|c|}
\hline \multicolumn{1}{|c|}{ Dataset } & Subtraction & $\mathbf{5 0 ~ I m a g e ~ A t l a s ~}$ & $\begin{array}{c}\text { PCA } \mathbf{~ m ( 5 0 )} \\
\text { Eigenimage Atlas }\end{array}$ & m Image atlas \\
\hline A.1 Left & 1.88 & 0.19 & 0.25 & 1.06 \\
\hline A.1 Right & 3.09 & 0.39 & 0.32 & 0.66 \\
\hline A.2 Left & 2.70 & 0.53 & 0.67 & 0.77 \\
\hline A.2 Right & 1.24 & 0.26 & 0.29 & 1.01 \\
\hline A.3 Left & 2.30 & 0.30 & 0.44 & 0.36 \\
\hline A.3 Right & 0.79 & 0.14 & 0.17 & 0.33 \\
\hline Average Temperature Stability & $\mathbf{2 . 0 0}$ & $\mathbf{0 . 3 0}$ & $\mathbf{0 . 3 6}$ & $\mathbf{0 . 7 0}$ \\
\hline
\end{tabular}

Figure 5: Temperature Stability: this graph reports the temporal standard deviation of the spatial average of the temperature within a selected ROI for 6 kidney motion datasets. The comparison displays that a PCA condensed atlas (green) achieves similar correction to the full 50 image atlas (red). Using an atlas with the same number of images as the PCA atlas results in limited correction due to the short observation period (purple). The PCA atlas provides effective correction while minimizing atlas size and computational cost.

Compared with subtraction, the average improvement achieved using each method is as follows:

○ $1.70{ }^{\circ} \mathrm{C}$ for the 50 image atlas (method 2$)$

$\circ \quad 1.64{ }^{\circ} \mathrm{C}$ for the PCA $m(50)$ eigenimage atlas (method 3), and

○ $1.30{ }^{\circ} \mathrm{C}$ for the $m$ image atlas (method 4 )

This indicates that the condensed PCA representation can achieve nearly the same level of correction as the complete 50 image atlas. 
Temperature precision, the temporal average of the spatial standard deviation, is also shown below.

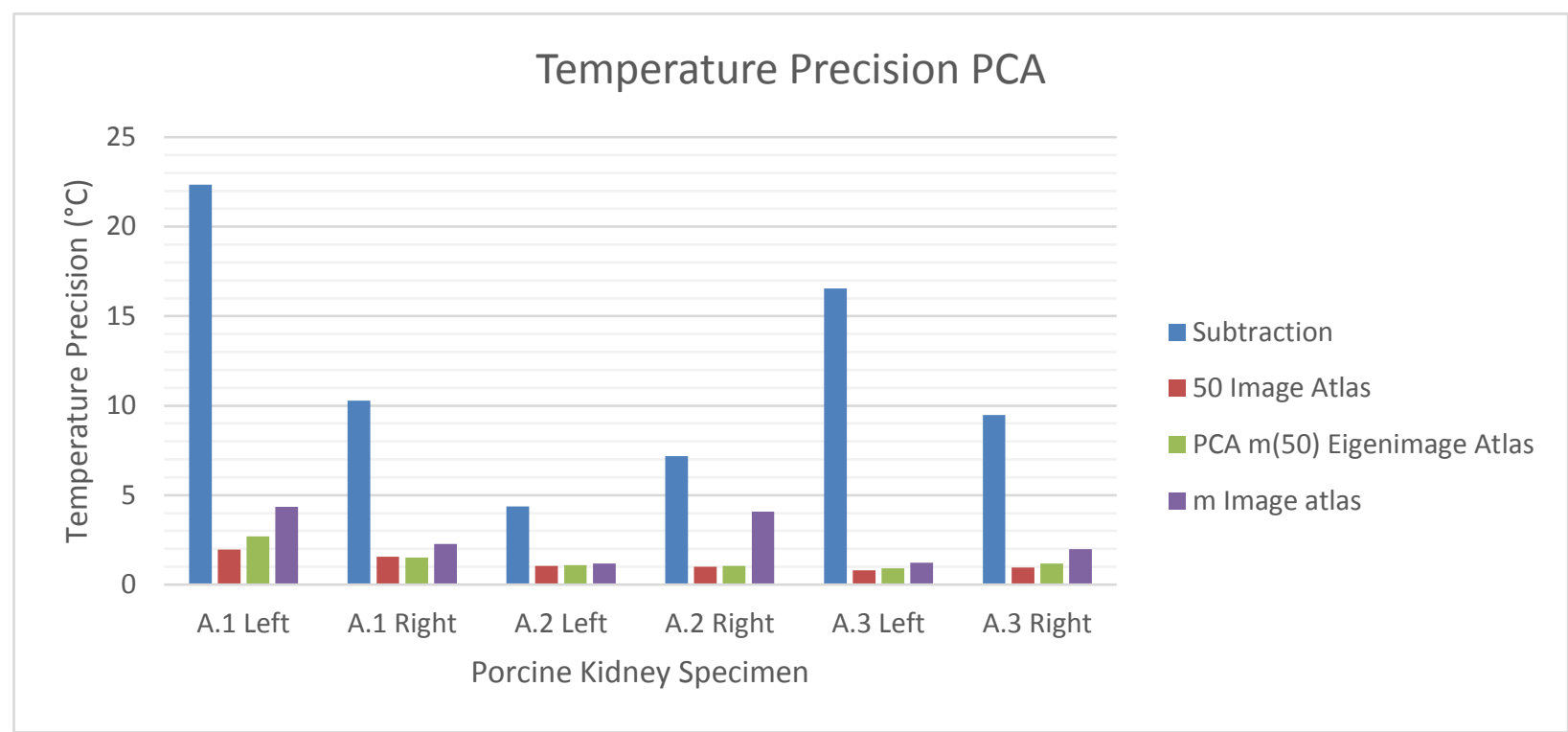

\begin{tabular}{|l|c|c|c|c|}
\hline \multicolumn{1}{|c|}{ Dataset } & Subtraction & 50 Image Atlas & $\begin{array}{c}\text { PCA m(50) } \\
\text { Eigenimage Atlas }\end{array}$ & m Image atlas \\
\hline A.1 Left & 22.34 & 1.97 & 2.70 & 4.35 \\
\hline A.1 Right & 10.28 & 1.56 & 1.52 & 2.27 \\
\hline A.2 Left & 4.36 & 1.04 & 1.09 & 1.18 \\
\hline A.2 Right & 7.17 & 1.01 & 1.04 & 4.08 \\
\hline A.3 Left & 16.55 & 0.80 & 0.91 & 1.23 \\
\hline A.3 Right & 9.47 & 0.96 & 1.18 & 1.99 \\
\hline Average Temperature Precision & $\mathbf{1 1 . 7 0}$ & $\mathbf{1 . 2 2}$ & $\mathbf{1 . 4 1}$ & $\mathbf{2 . 5 2}$ \\
\hline
\end{tabular}

Figure 6: Temperature Precision: this graph reports the temporal average of the spatial standard deviation of the temperature within a selected ROI for 6 kidney motion datasets. The PCA atlas uses a condensed representation of $m$ eigenimages derived from 50 images; performance is similar to the complete set of 50 images with only the computational demands of the $m$ image atlas.

For temperature precision, the average improvement compared with subtraction is:
○ $10.47^{\circ} \mathrm{C}$ for the 50 image atlas (method 2)
○ $10.29{ }^{\circ} \mathrm{C}$ for the PCA $m(50)$ eigenimage atlas (method 3), and
○ $\quad 9.18{ }^{\circ} \mathrm{C}$ for the $m$ image atlas (method 4$)$ 
B) Sonication dataset

Figure 7 and Figure 8 display results from the sonication dataset. Each graph plots the temperature within a ROI for the entire duration of the procedure. The standard deviation of the temperature is evaluated in the steady heating portion annotated in the graphs.

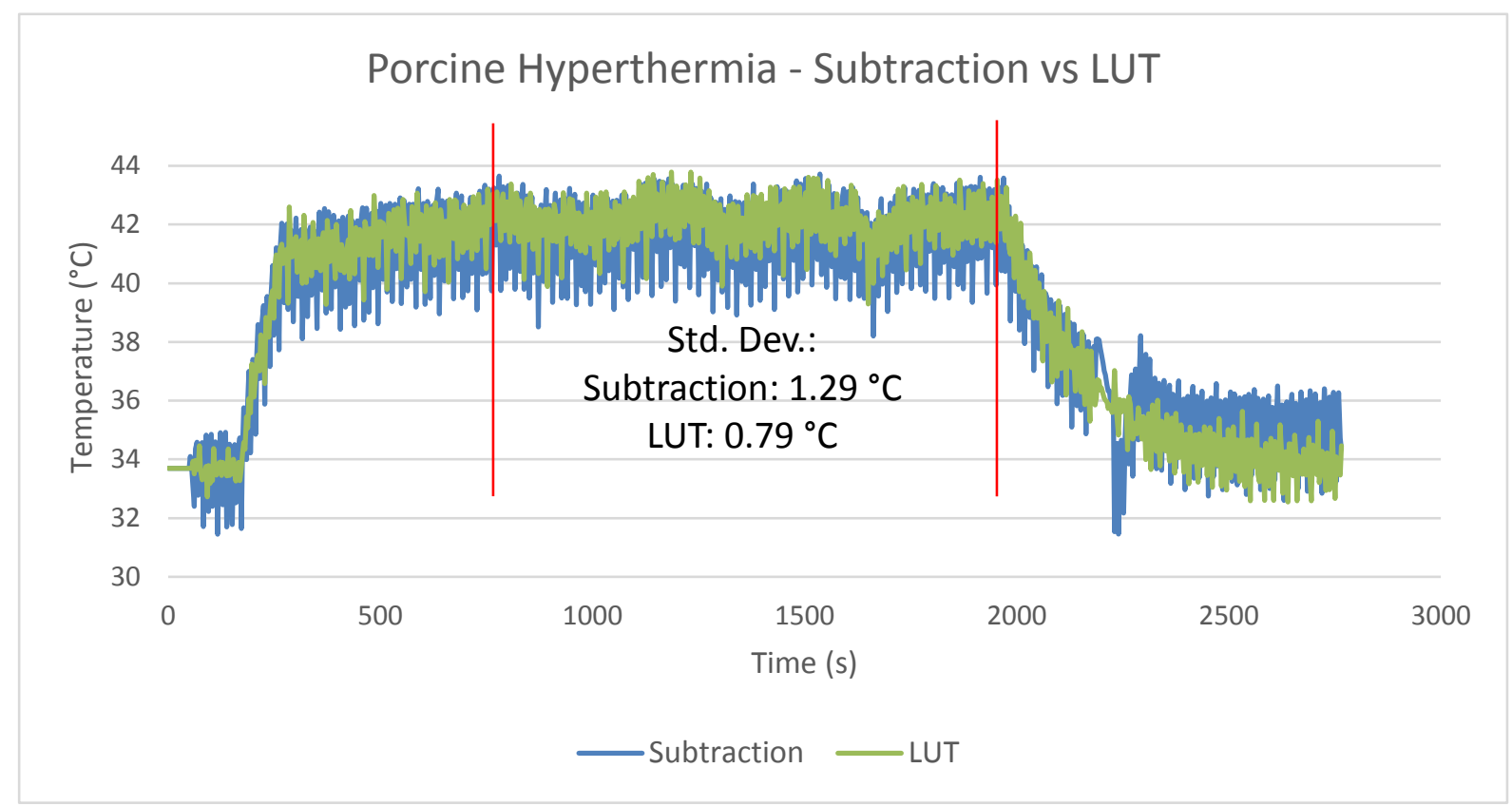

Figure 7: Hyperthermia thermometry via standard subtraction and navigator echo LUT. The navigator echo LUT method achieves a $39 \%$ improvement compared to subtraction. 


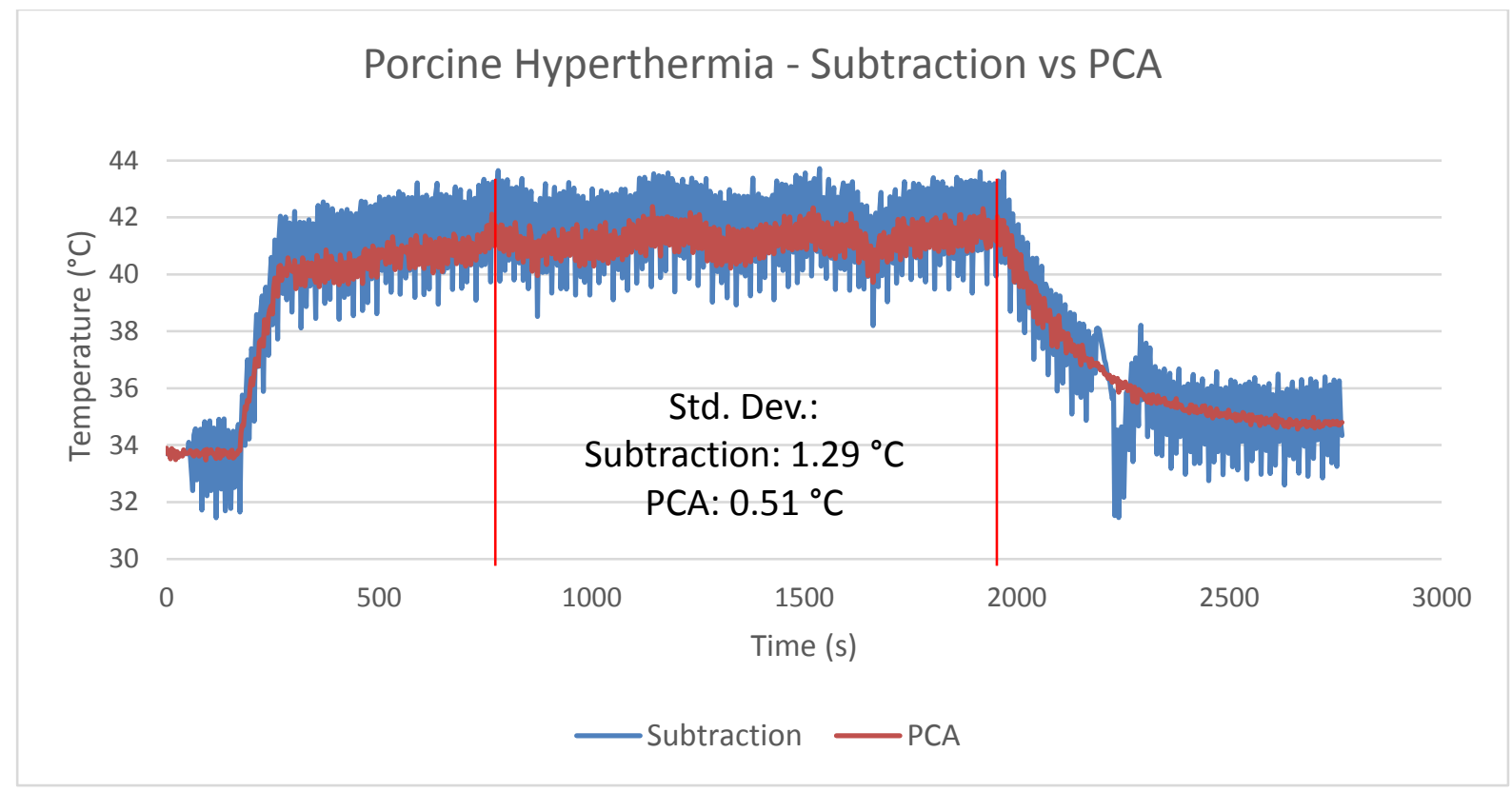

Figure 8: Hyperthermia thermometry via standard subtraction and PCA projection. The PCA method achieves a $60 \%$ improvement compared with subtraction and a $35 \%$ improvement compared to the navigator echo LUT method.

Both the LUT and PCA achieve artifact reduction. Qualitatively, PCA is able to report a more steady temperature throughout the procedure than the other methods. The difference is most evident in the cooldown period, starting at about $2000 \mathrm{sec}$. In the navigator echo LUT method, this cooldown period appears to have the same level of variance as the period of manually controlled hyperthermia. Whereas in the PCA method, the descent is more stable, which may indicate that the heating introduces some variance due to the manual control. Note that the standard deviation during this heating is still well under $1^{\circ} \mathrm{C}$. Within this heating period, PCA achieves the lowest standard deviation of all the methods. Compared with the navigator echo LUT method, PCA provides a $0.28{ }^{\circ} \mathrm{C}$ reduction in standard deviation (35\% improvement) without the use of any supplementary imaging or tracking tools.

\subsection{PCA Discussion}

PCA offers robust correction of periodic artifacts across all datasets. PCA also allows for longer observation periods while reducing data storage and computational costs. A longer period of observation increases the probability that the atlas will closely match the phase patterns in the new dynamic image. However, long observation periods also include more redundant data, 
particularly if the artifact repetition period is shorter than the observation period. The PCA method calculates and keeps only the principal components of the data, creating an atlas that exploits long observation periods while reducing redundancy in the data. This is important because the length of the artifact period is unpredictable. The same dataset used in the eigenvector analysis (Figure 1) exhibits repeating artifacts with a long period. As seen in Figure 9, this artifact's period is clearly longer than the respiratory period of the porcine subject which is typically less than a few dynamics. This could be caused by under-sampling because the dynamic scan time is only slightly shorter than the respiratory cycle. If the dynamic scan time is close to the period of the respiratory cycle it can take many dynamics before the entire period is sampled. In contrast, another dataset exhibits an artifact with a very short period.

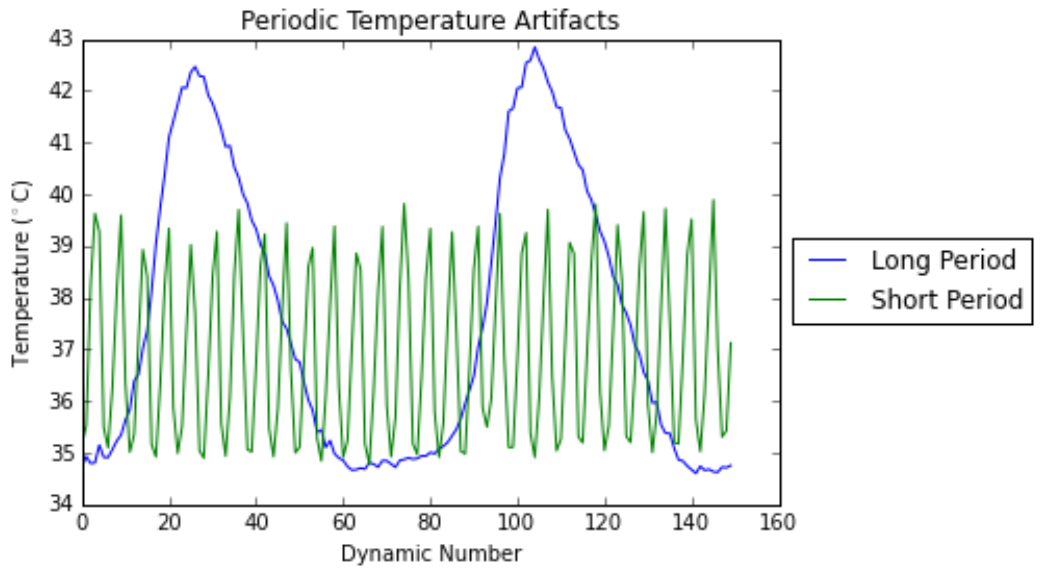

Figure 9: Periodic artifacts with a long (blue) and short (green) periods from two different porcine subjects. Because the period length can be unpredictable, having a longer observation period increases the probability that the atlas will sample a larger portion of the period.

As such, a longer observation period makes the correction more robust against unpredictable artifact periods.

PCA's corrective ability is also demonstrated in the temperature stability and precision analysis. For both metrics, PCA scored closer to the full 50 image atlas method than to the $m$ image atlas method. This demonstrates PCA's ability to retain the most useful components of the data within a condensed representation. Compared with the $m$ image atlas method, PCA's accuracy advantage is quite modest; however, PCA's most valuable aspect is the deliberate design behind the atlas construction. This helps improve robustness and control as opposed to the $m$ image 
atlas method which simply uses the first $m$ images, regardless of how representative they are of the subject's patterns.

It is also important to note that even data with low temperature stability and precision scores can severely impair thermometry monitoring. The thermometry from the A. 2 Left Kidney specimen has relatively low temperature stability and precision even for the subtraction method, $2.70{ }^{\circ} \mathrm{C}$ and $4.36{ }^{\circ} \mathrm{C}$, respectively (data presented in Figure 5 and Figure 6). However, Figure 10 displays artifacts in this dataset that could seriously impact the accuracy of a procedure. Even artifacts in the periphery, which do not directly affect the accuracy of the treatment zone, can make it difficult for health care providers to confidently proceed and ensure patient safety. 

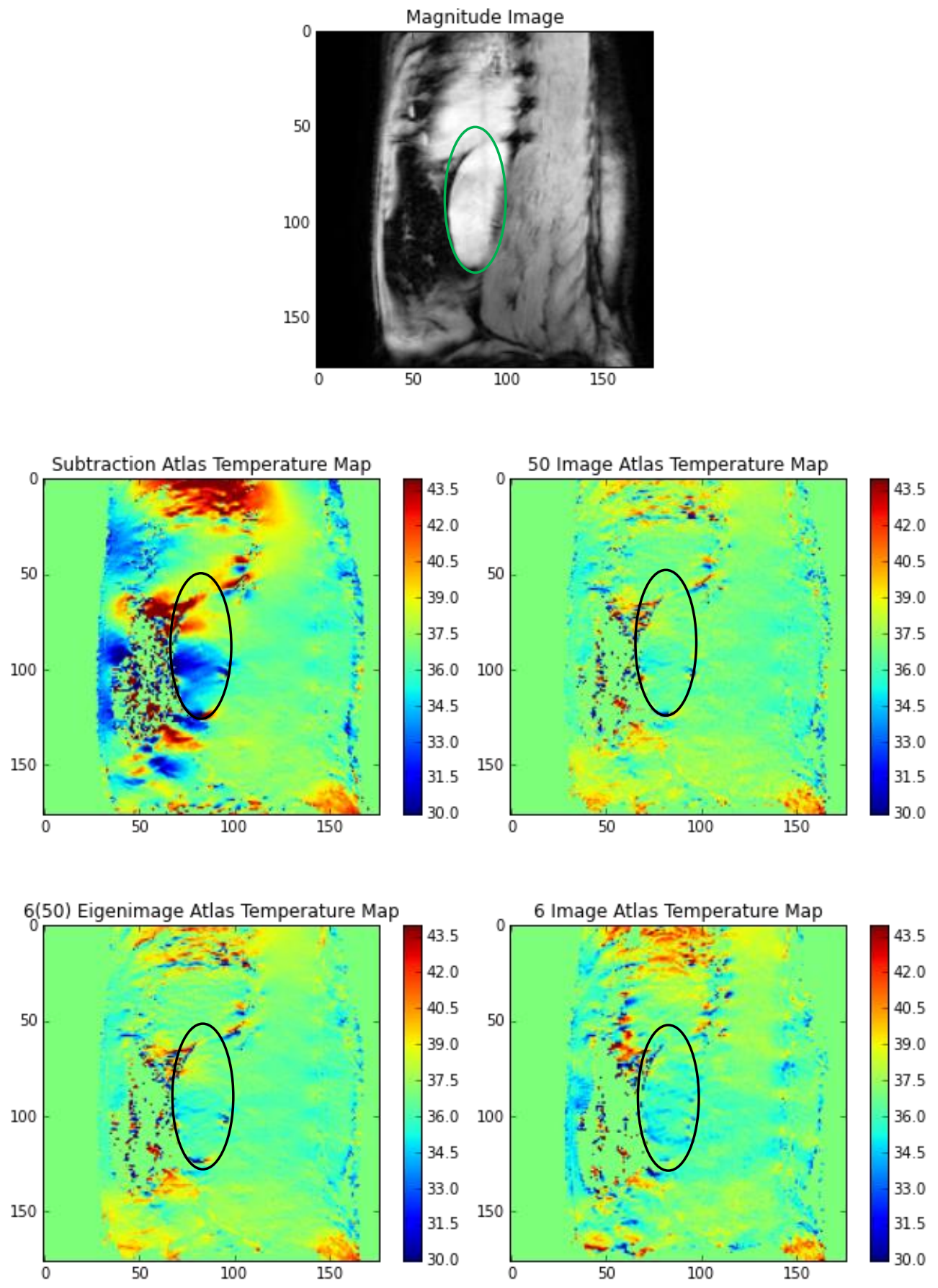

Figure 10: (Top) magnitude image for reference, (bottom) temperature maps with severe artifacts despite low temperature stability and precision. Even at low intensities, widespread artifacts can obscure hotspots and add uncertainty to the condition of surrounding tissue.

As such, seemingly marginal improvements in temperature stability and precision can still greatly impact the feasibility of a procedure. 
While PCA achieves a good compromise between computational time and correction accuracy, the computational demands of the full atlas (method 2) are still well under the dynamic scan time. The average computation time for the 50 image atlas in method 2 is $152 \mathrm{~ms}$, which is only about one quarter of the dynamic scan time for a single image $(573 \mathrm{~ms})$. Nevertheless, PCA is able to reduce this computation time to roughly $26 \mathrm{~ms}$, while still accounting for over $99.9 \%$ of the variance within the observation period. The speed advantage of PCA will become increasingly important as image size increases and dynamic scanning time decreases. Both of these trends are converging in efforts to develop real-time 3D thermal imaging. As such, this method's benefits will continue to grow as imaging techniques improve.

The sonication dataset illustrates the advantage of using projection-based methods over motion tracking tools. Comparison with navigator look-up-table method demonstrates improved temperature stability without the need for any supplementary motion tracking tools or imaging. However, the PCA cool down period displayed in Figure 8 fails to return to the baseline temperature of $34^{\circ} \mathrm{C}$. This is likely due to slow magnetic field drift over the 45 minute procedure. The LUT method has two elements that help correct this field drift: i) LUT updates and ii) drift correction.

LUT updates allow the LUT atlas itself to be periodically updated using newly acquired images. This fills the LUT atlas with reference images that more closely resemble the current state of the phase. This can be extremely advantageous, particularly if the tissue shifts to a new position. Note that adding new images to the atlas is typically not acceptable because new phase images may already contain heat-induced phase changes. As such, this LUT method tracks the temperature change history associated with each reference image so that no heat-induced phase change is lost. Unfortunately this is not trivial to implement within the PCA method because PCA reference images are decomposed into eigenimages. This makes it very difficult to associate a temperature history with a particular eigenimage.

The other element that helps with field drift is 2D drift correction. This is accomplished by using a $2^{\text {nd }}$ order polynomial fit scheme which models low spatial frequency phase changes caused by field drift. This can be added to PCA, however the PDF method presented in the following chapter is able to achieve similar correction. 
Overall, the major drawback of PCA is its inability to correct aperiodic artifacts. This is an inherent weakness of nearly all multi-baseline methods and the rationale for the development of the PDF method presented in the following chapter. 


\section{Chapter 3 \\ Projection onto Dipole Fields: Correction of Aperiodic Susceptibility Artifacts}

\subsection{Theory behind using PDF as a Near-Referenceless Method}

Correcting for aperiodic artifacts from both motion and susceptibility changes requires an algorithm that can synthesize a range of naturally occurring phase patterns. Most critically, the algorithm must also be able to discriminate between natural phase patterns and heat-induced phase effects. This means referenceless methods must accurately model all physical characteristics of the magnetic field, excluding effects caused by heating.

Low spatial frequency variations of largely homogenous magnetic fields can be easily modeled with smooth low-order polynomials. In contrast, high spatial frequency features within the magnetic field can be difficult to distinguish from the hotspots created via HIFU. This is why multi-baseline methods are typically used to correct for anatomical features that cause inhomogeneity (see phase image in Figure 2). Much of the inhomogeneity stems from magnetic susceptibility differences between the tissue and other nearby matter, most notably bone or air. Air in particular is prone to aperiodic changes. The high spatial frequency and aperiodic nature of air-related susceptibility artifacts makes them difficult to correct with either multi-baseline or referenceless approaches.

The proposed PDF method is adapted from quantitative susceptibility mapping [29], where it is used for removal of background susceptibility effects. All materials in the body have a magnetic susceptibility that impacts the magnetic field. The effect on the phase is seen when there is a relative difference in susceptibility. PDF is used to model the effects of these susceptibility differences on the phase. More specifically, it is used to determine a susceptibility distribution that induces a given phase pattern. Note that a given pixel can be assigned a magnetic susceptibility value based on the material contained in that space. However, the phase value at a given pixel is a superposition of all nearby susceptibility distortions of the field. For example, regions of air or bone can be clearly delineated in magnitude images, however in the phase images they produce a wider area of effect which is not as easily demarcated (see Figure 2). 
The conversion from phase pattern to susceptibility distribution is very useful because it provides a representation in which different contributing factors to the phase can be isolated. The aim is to use this method to remove the aperiodic artifacts associated with air or bone regions that are difficult to model with existing thermometry correction strategies

\subsection{PDF Method}

PDF method was first developed for quantitative susceptibility mapping [29]. It is used to model and remove susceptibility artifacts from background materials such as bone and air. As mentioned in Section 3.1, the bone and air regions can be clearly delineated in the magnitude images and PDF provides a way to determine the phase effects associated with these regions. These background regions are automatically determined for each dynamic by calculating an SNR mask based on the magnitude of the new dynamic, the PCA generated reference, and a masking threshold.

The relationship between the magnetic susceptibility in these background regions and their phase effects is calculated through a dipole model. The dipole model chosen for this thermometry application is the cylindrical dipole model (3.1):

$$
d=\frac{Y^{2}-X^{2}}{\left(X^{2}+Y^{2}\right)^{2}}(3.1)[30],[31]
$$

This 2D model is used to fit typical thermometry acquisition protocols which are done in 2D. The original PDF method's 3D dipole model would require about 11 slices and would have a dynamic scan time of about $8.6 \mathrm{sec}$ using similar scan parameters. This was used in preliminary phantom and ex vivo studies for proof-of-concept, but to monitor critical in vivo procedures this is infeasible. During clinical procedures, it is important to have a short dynamic scan time to accurately monitor the temperature elevation. A single sonication can reach ablative temperatures $\left(56-100^{\circ} \mathrm{C}\right)$ within 1-3 seconds [32]. As such, a high temporal resolution is important to accurately track the temperature evolution. Also, within 8.6 seconds, different slices in the abdomen can become misaligned with non-rigid differences. This would violate the continuity of the 3D data and invalidate the use of the 3D dipole model. 
The conversion between susceptibility and phase is approximated through convolution with this 2D dipole model. Performing this computation as a multiplication in the Fourier domain gives a simplified and more manageable format of $A x=b$ :

$$
D \chi=\phi(3.2)
$$

Where $D$ is the 2D dipole model, $\chi$ is the susceptibility distribution within the background regions, and $\phi$ is the residual phase after the subtraction of the PCA reference.

Using PDF as a 2D scheme, it is not possible to completely model the entire phase image which is actually a result of the $3 \mathrm{D}$ environment of the body. Instead, PCA is leveraged to take care of the majority of the phase effects, to the extent of what is known to the atlas. As such, PDF is performed after PCA and only tries to model the residual phase patterns, $\Delta \phi_{P C A}$ (see Section 2.2), that exist after the PCA reference has been subtracted from the new dynamic. This order of PCA followed by PDF is chosen to best exploit the phase data that has already been captured in the atlas. This post-PCA residual may include (aperiodic) background artifacts as well as heating, but the PDF model will only fit background susceptibility artifacts. Heat-induced phase patterns will remain unchanged because the dipoles in the background region will have limited ability to recreate a hotspot within the tissue region. This is validated in the paper by Liu et al., 2011 [29], which indicates that the maximum absolute normalized inner product between any local dipole and any background dipole is approximately zero, except within about 5 pixels from the tissue border [29]. This means the local and background dipole fields are approximately orthogonal. The same principle is applied in the original use of PDF, in quantitative susceptibility mapping, to preserve susceptibility effects within the tissue, e.g. iron depositions or calcium in the brain. Note that this method is not capable of correcting artifacts that originate within the tissue; the similarity between such artifacts and actual heating is too close to discount such artifacts with a high degree of certainty without additional information. An example of this is given in Section 3.4 .

The $A x=b$ format of the susceptibility equation (3.2) is efficiently solved using the conjugate gradient method. The conjugate gradient method can be used to minimize functions of the quadratic form: 


$$
f(x)=\frac{1}{2} x^{T} A x-b^{T} x+c(3.3)
$$

Graphically this can be thought of as a parabolic bowl shape. Thus the minimum can be found where the gradient is equal to zero:

$$
\begin{gathered}
f^{\prime}(x)=0(3.4) \\
\text { or } \\
\nabla f(x)=0 \text { (gradient) (3.5) }
\end{gathered}
$$

Conveniently, for symmetric A:

$$
\begin{gathered}
\nabla f(x)=A x-b=0(3.6) \\
A x=b(3.7)
\end{gathered}
$$

Thus finding the minimum of this function will also solve the $A x=b$ system. In this case, $A \equiv$ $D$ (2D dipole convolution), $x \equiv \chi$ (background susceptibility distribution), and $b \equiv \Delta \phi_{P C A}$ (residual phase).

This algorithm works by iteratively solving for $\chi$ by searching for the minimum of $f(x)$ along search directions that are $D$-orthogonal. Suppose $p$ is a set of $n$, (D-orthogonal) search directions that $\operatorname{span} \chi$. Then $\chi$ can be expressed as a sum of the distances traveled $(\alpha)$ along each search direction in $p$ :

$$
\chi_{*}=\sum_{i=0}^{n} \alpha_{i} p_{i} \text { where } \chi_{*} \text { is the solution to } D \chi=\Delta \phi_{P C A}
$$

This can be extended to:

$$
\Delta \phi_{P C A}=D \chi_{*}=\sum_{i=0}^{n} \alpha_{i} D p_{i}(3.9)
$$

Furthermore, because each $p_{i}$ is $D$-orthogonal to all other $p_{k}$ (where $k \neq i$ ), then $p_{k} D p_{i}=0$ :

$$
\begin{gathered}
\triangle \phi_{P C A}=D \chi=\sum_{i=0}^{n} \alpha_{i} D p_{i}(3.10) \\
p_{k}^{T} \Delta \phi_{P C A}=\sum_{i=0}^{n} \alpha_{i} p_{k}^{T} D p_{i} \\
p_{k}^{T} \Delta \phi_{P C A}=\alpha_{k} p_{k}^{T} D p_{k}(3.12) \\
\alpha_{k}=\frac{p_{k}^{T} \Delta \phi_{P C A}}{p_{k}^{T} D p_{k}}(3.13)
\end{gathered}
$$


In this way it is easy to solve for $\alpha_{i}$, the distance traveled along search direction $p_{i}$, that will minimize $f(x)$. As an iterative method, each new search direction $p_{i}$ is determined sequentially based on the residual and existing $p_{j}$ (where $j<i$ ).

Once the background susceptibility distribution, $\chi_{\text {background }}$, is determined, $\phi_{\text {background }}$ can be calculated as

$$
\text { D } \chi_{\text {background }}=\phi_{\text {background }}(3.14)
$$

Where $\phi_{\text {background }}$ is the PDF approximation for $\Delta \phi_{P C A}$, which models only background susceptibility artifacts. The heating can then be calculated as

$$
\Delta \phi_{\text {heat }}=\Delta \phi_{P D F}=\Delta \phi_{P C A}-\phi_{\text {background }}
$$

This method provides a convenient way to calculate and remove the phase effects of all background dipoles simultaneously.

\subsection{Experiment Method and Specifications}

\subsubsection{Scan Parameters}

This chapter uses the same datasets (A and B) described in the PCA chapter. Refer to Section 2.3.1 for a list of the scan parameters used.

C) Human volunteer datasets

This chapter analyzes additional datasets of human volunteers under free breathing. Imaging was performed on Philips 3T Achieva scanner using the following parameters: FOV: 300 x $300 \mathrm{~mm}$, voxel size $=1.34 \mathrm{~mm}$, slice thickness $=11 \mathrm{~mm}, \mathrm{TE} / \mathrm{TR}=16 / 26 \mathrm{~ms}$, flip angle $=20^{\circ}$, acquisition matrix $=200 \times 198$, reconstruction matrix $=224, \mathrm{ETL}=9, \mathrm{NEX}=1$, slices $=1$, dynamic time $=$ $0.58 \mathrm{~s}$. This dataset examines the artifacts present in the human abdomen. This verifies the method in human anatomy as compared with porcine datasets which exhibit different anatomy, patient positioning, and breathing patterns. Porcine subjects were positioned decubitus/supine while human volunteers were completely supine. The difference in positioning may also lead to 
the observation of different motion patterns. Furthermore, human volunteers were not ventilated or sedated, producing free breathing patterns.

\subsubsection{Data Processing}

Note that all PDF processing is performed after initial PCA processing.

A) Kidney motion datasets

Out of the 6 kidney motion datasets analyzed using PCA, 3 were selected for PDF processing. The other 3 datasets did not exhibit any aperiodic artifacts.

For comparison, a re-weighted $6^{\text {th }}$ order polynomial fit scheme was extended from the $2^{\text {nd }}$ order drift correction method in [19]. The polynomial fit processing is performed after identical PCA processing. Note that this PCA processing is done using the $m$ image atlas method, meaning there is no dimensionality reduction. This makes the PCA portion mathematically equivalent to a standard projection method. As such, this PCA-Polynomial Fit benchmark mimics the hybrid method described in [22] and Section 1.2.4.

B) Sonication dataset

As mentioned in the PCA discussion (Section 2.5), PDF is able to approximate the artifacts associated with slow magnetic field drift. This is tested by processing the sonication dataset using PCA-PDF. The PCA portion of this processing is identical to the previous PCA processing (Section 2.3.2).

C) Human volunteer datasets

The human volunteer datasets were processed using PCA-PDF. This is compared with processing via the PCA-Polynomial Fit benchmark. Note PCA processing is identical in both cases.

Furthermore, a hotspot from a phantom experiment was superimposed onto one of the kidneys exhibiting severe aperiodic artifacts. This tests PDF's ability to discriminate between heating and artifacts. This data was then processed using PCA-PDF. In this way, the hotspot data provides a gold standard for the desired temperature. 


\subsection{PDF Results}

\section{A) Kidney motion datasets}

Below (Figure 11) are standard deviation maps from one of the datasets. These display PDF and polynomial fit correction compared with the PCA correction that precedes both methods.
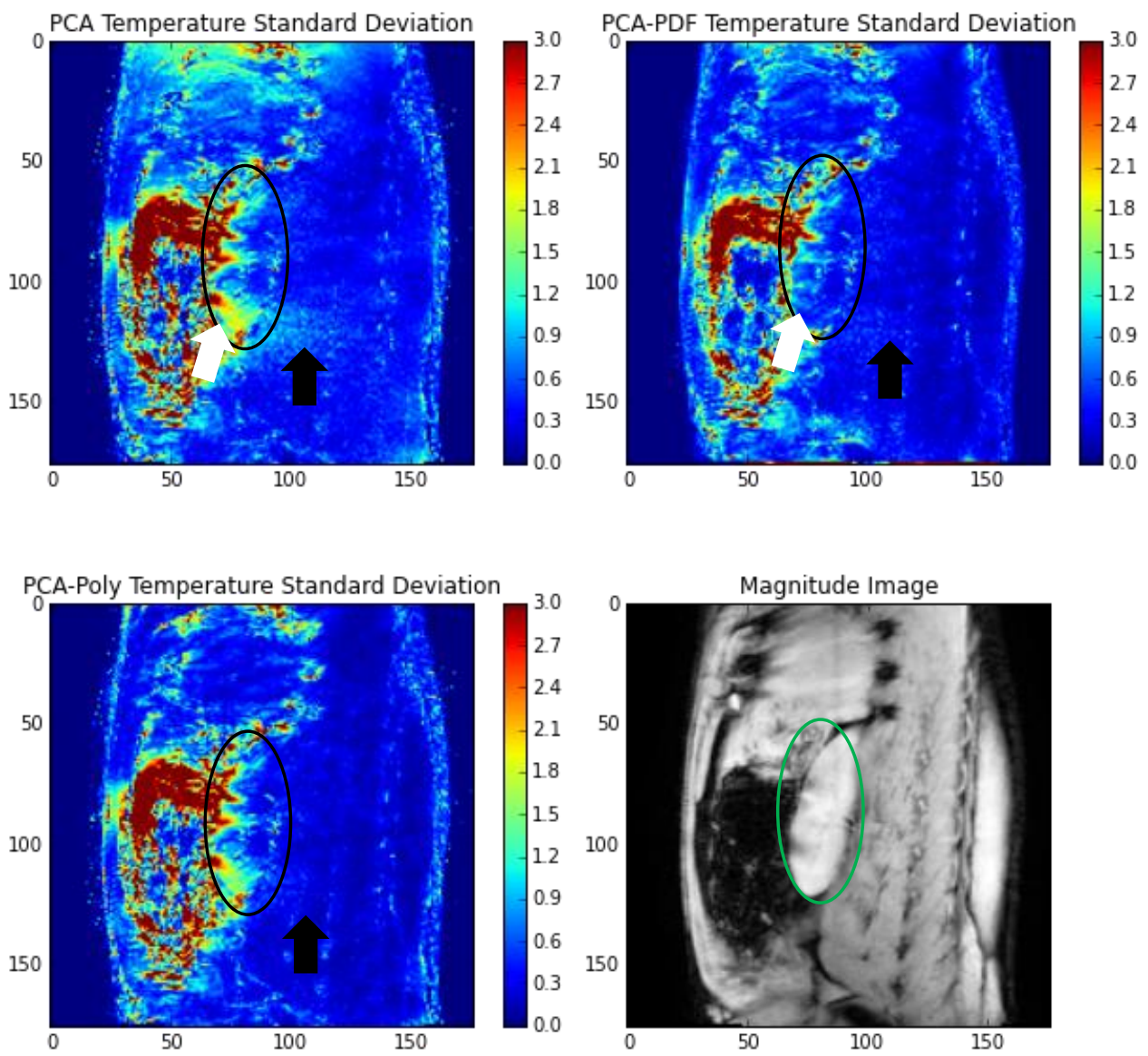

Figure 11: Left kidney temperature standard deviation maps, and magnitude image (bottom right) for reference. Artifacts that are not recorded in the atlas appear in the PCA map (top left). PCA-PDF (top right) provides correction for sharp (white arrow) and smooth (black arrow) artifacts near the bottom of the kidney, while PCA-Polynomial fit (bottom left) is only able to reduce the smooth artifacts (black arrow).

There is little added correction achieved through polynomial fit. This can be seen more clearly in the temporal plot of the temperature within an ROI in the kidney (Figure 12). Note that this artifact is periodic, but was not captured within the atlas observation period. As such, it is considered an unrecorded phase change that must be corrected using a referenceless method. 


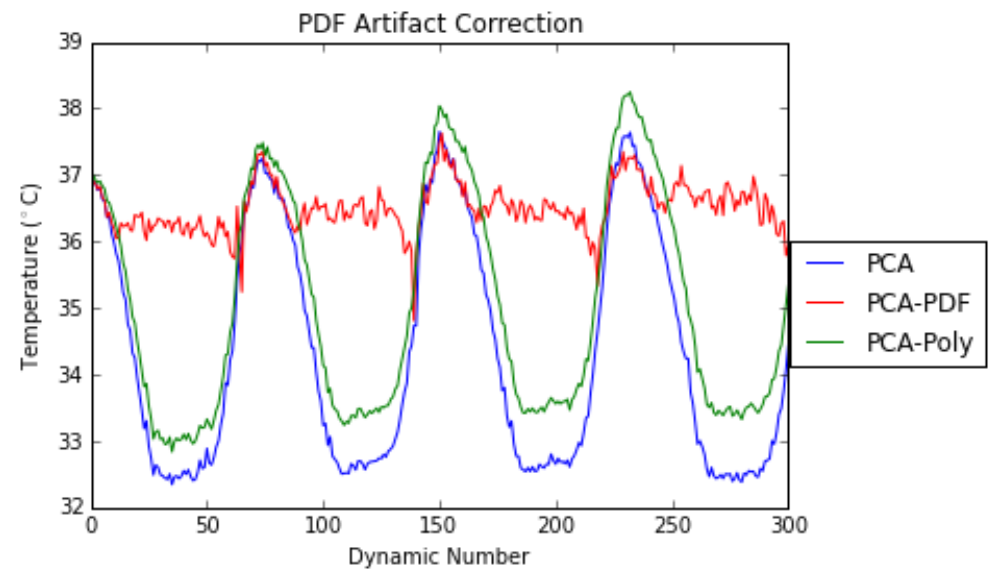

Figure 12: Artifact correction using PDF. PCA-PDF (red) removes the majority of the original artifact (blue). In contrast, PCA-Polynomial fit only dampens the severity by removing the smooth, low spatial frequency component of the artifacts.

The PCA-Polynomial Fit method generally dampens the severity of the artifact, but largely fails to remove it. This is because the artifact, caused by a susceptibility change at the tissue boundary, contains both low and high spatial frequency components. Polynomial fit is only designed to model low spatial frequency components, while PDF is capable of removing artifacts which fit the dipole model so long as the source is at the tissue boundary.

Temperature stability and precision are also reported in the graphs below (Figure 13 and Figure 14). In both cases, PDF performs substantially better than polynomial fit for the first two datasets (left kidneys), but does not reduce the artifacts in the third dataset (right kidney). 


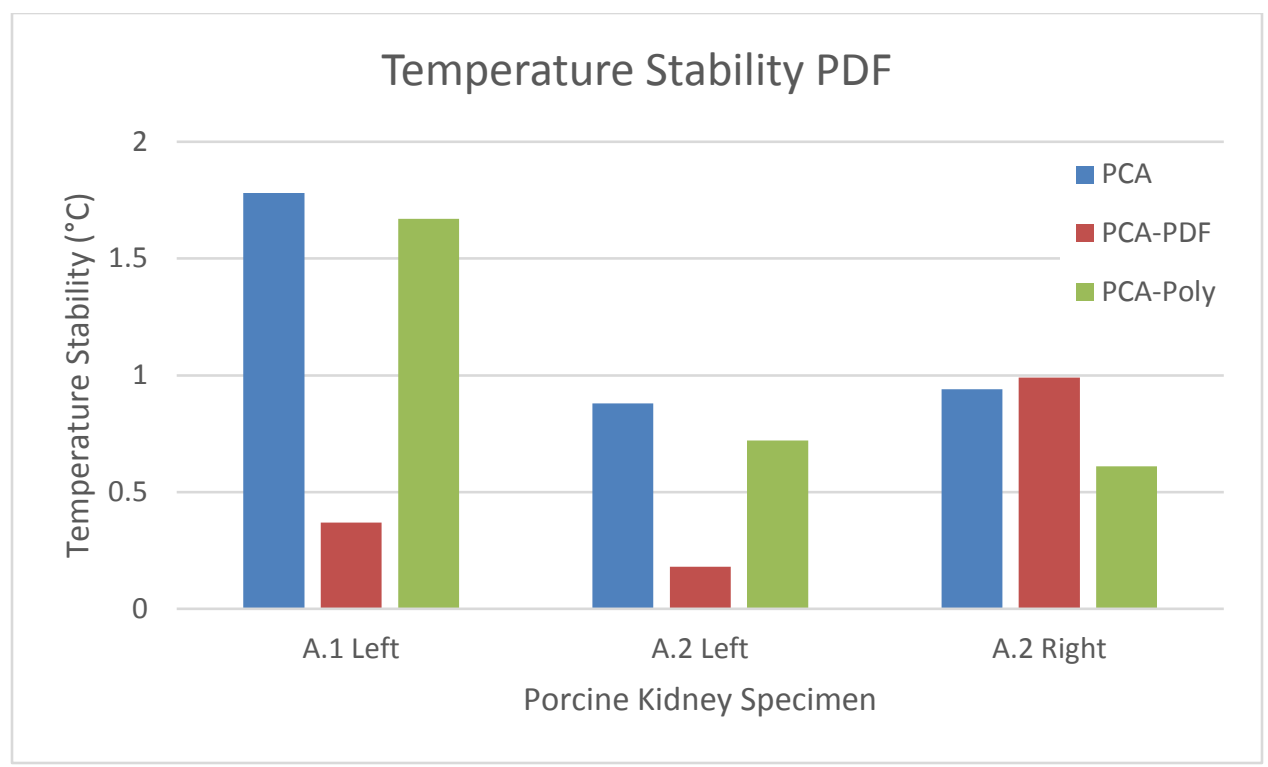

\begin{tabular}{|l|c|c|c|}
\hline \multicolumn{1}{|c|}{ Dataset } & PCA & PCA-PDF & PCA-Poly \\
\hline A.1 Left & 1.78 & 0.37 & 1.67 \\
\hline A.2 Left & 0.88 & 0.18 & 0.72 \\
\hline A.2 Right & 0.94 & 0.99 & 0.61 \\
\hline Average Temperature Stability & $\mathbf{1 . 2 0}$ & $\mathbf{0 . 5 1}$ & $\mathbf{1 . 0 0}$ \\
\hline
\end{tabular}

Figure 13: Temperature stability: this graph reports the temporal standard deviation of the spatial average of the temperature within a selected ROI for 3 kidney motion datasets. PCA-PDF is only able to correct the edge artifacts in the left kidney datasets and not the internal artifact in the right kidney dataset. 


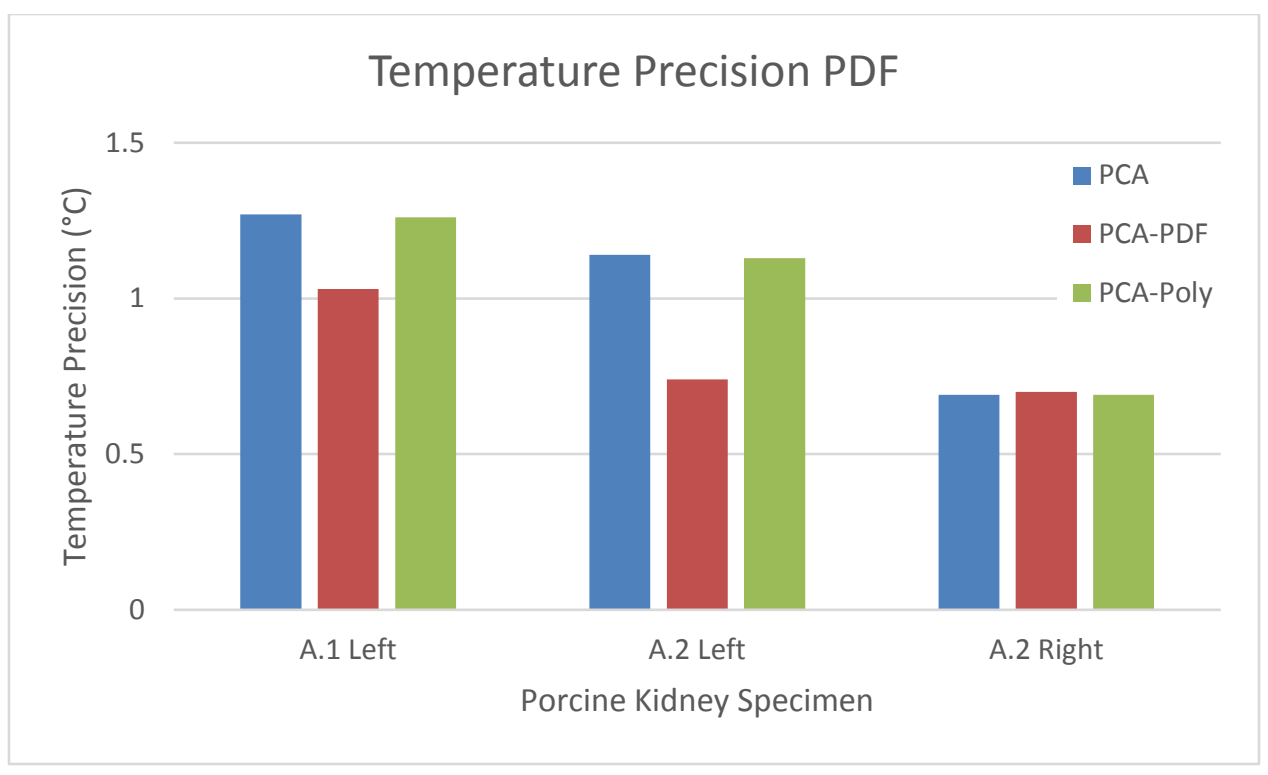

\begin{tabular}{|l|c|c|c|}
\hline \multicolumn{1}{|c|}{ Dataset } & PCA & PCA-PDF & PCA-Poly \\
\hline A.1 Left & 1.27 & 1.03 & 1.26 \\
\hline A.2 Left & 1.14 & 0.74 & 1.13 \\
\hline A.2 Right & 0.69 & 0.70 & 0.69 \\
\hline Average Temperature Precision & $\mathbf{1 . 0 3}$ & $\mathbf{0 . 8 2}$ & $\mathbf{1 . 0 3}$ \\
\hline
\end{tabular}

Figure 14: Temperature precision: this graph reports the temporal average of the spatial standard deviation of the temperature within a selected ROI for 3 kidney motion datasets. PCA-PDF only helps to improve the edge susceptibility artifacts in the left kidney datasets.

The key difference between the left and right kidneys is the surrounding anatomy. The magnitude image in Figure 11 indicates that the left kidney is adjacent to an area of low signal, likely composed of inhomogeneous substances associated with the bowel. In contrast, the right kidney (Figure 15) is tightly packed between relatively homogenous tissues. The low standard deviation in the right kidney is a direct result of this uniform environment. In fact, the standard deviation is so low that there is no meaningful difference within the kidney. As such, a ROI was selected in the tissue adjacent to the kidney (see annotated circle in Figure 15) for the temperature stability and precision calculations. 

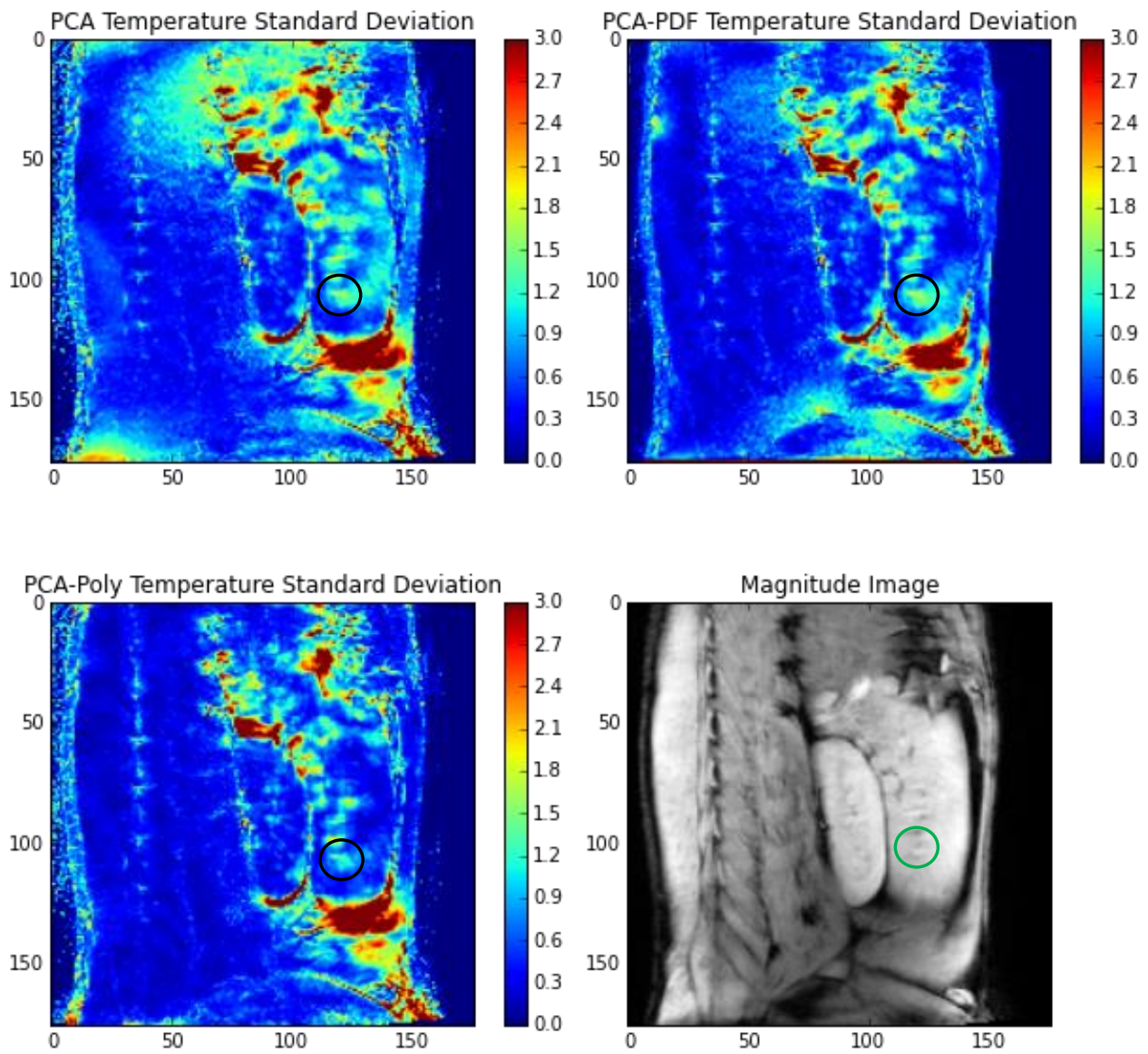

Figure 15: Right kidney temperature standard deviation maps and magnitude image (bottom right) for reference. PCA-PDF is not able to reduce this internal artifact (marked by the ROI). PCAPolynomial fit achieves a visible reduction.

Polynomial fit is able to reduce the low spatial frequency artifacts appearing within the center of the tissue, while PDF is not. This may make polynomial fit seem advantageous, however discounting phase changes in the center of the tissue as artifacts may be incorrect in certain circumstances. Figure 16 displays the temporal evolution of this artifact in the center of the tissue. This artifact reaches almost $40{ }^{\circ} \mathrm{C}$, which is very close to the $41{ }^{\circ} \mathrm{C}$ target temperature in the hyperthermia sonication dataset. The similarities between this artifact and a diffuse hyperthermia procedure make it difficult to distinguish between the two with a high degree of certainty. 


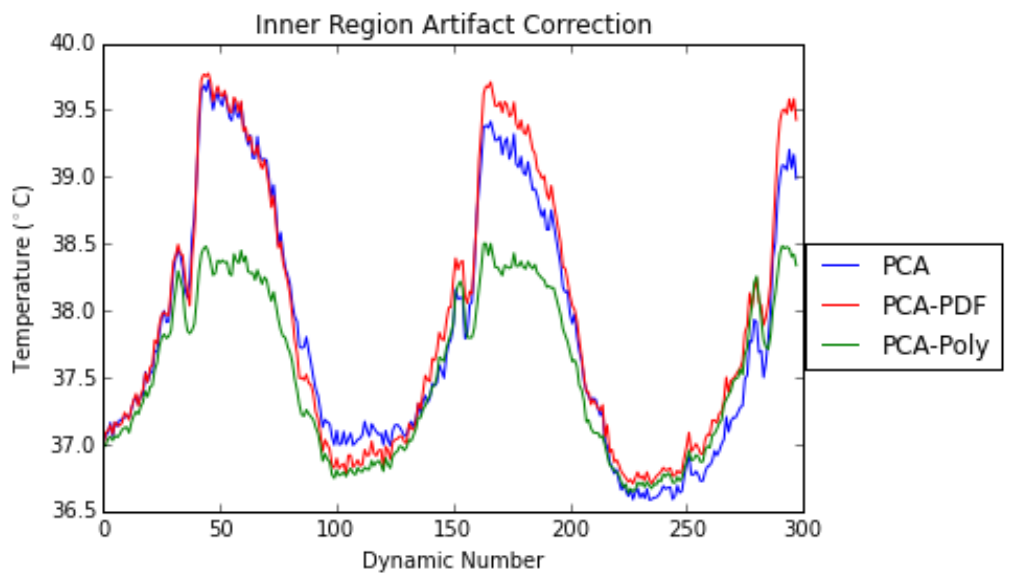

Figure 16: Artifact in the center of the tissue: PCA-Polynomial fit reduces the severity of this internal, low-intensity and diffuse artifact. This correction may also dampen true internal heating with similar phase characteristics, i.e. diffuse hyperthermia.

B) Sonication dataset

The graph in Figure 17 demonstrates PDF's ability to model global magnetic field drift by approximating the gradual phase changes using background dipoles. Here the temperature is returned to the $34{ }^{\circ} \mathrm{C}$ baseline. 


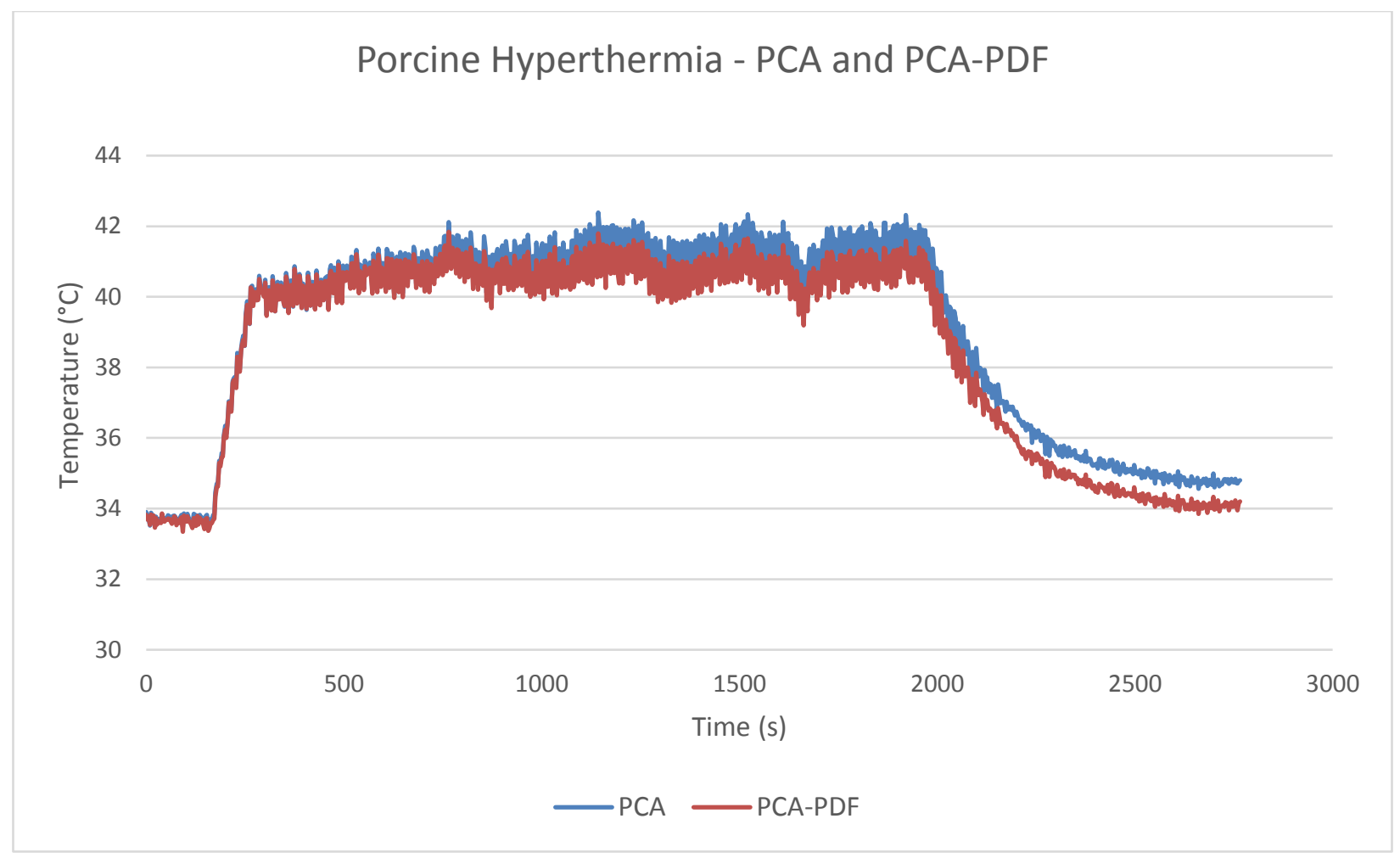

Figure 17: Drift correction using PDF: The addition of PDF aids in removing gradual magnetic field drift. Note that this gradual field drift is due to temperature changes in the MR hardware over the duration of the scan [8].

\section{C) Human volunteer datasets}

Temperature stability and precision are shown for the two human volunteers in Figure 18 and Figure 19. 


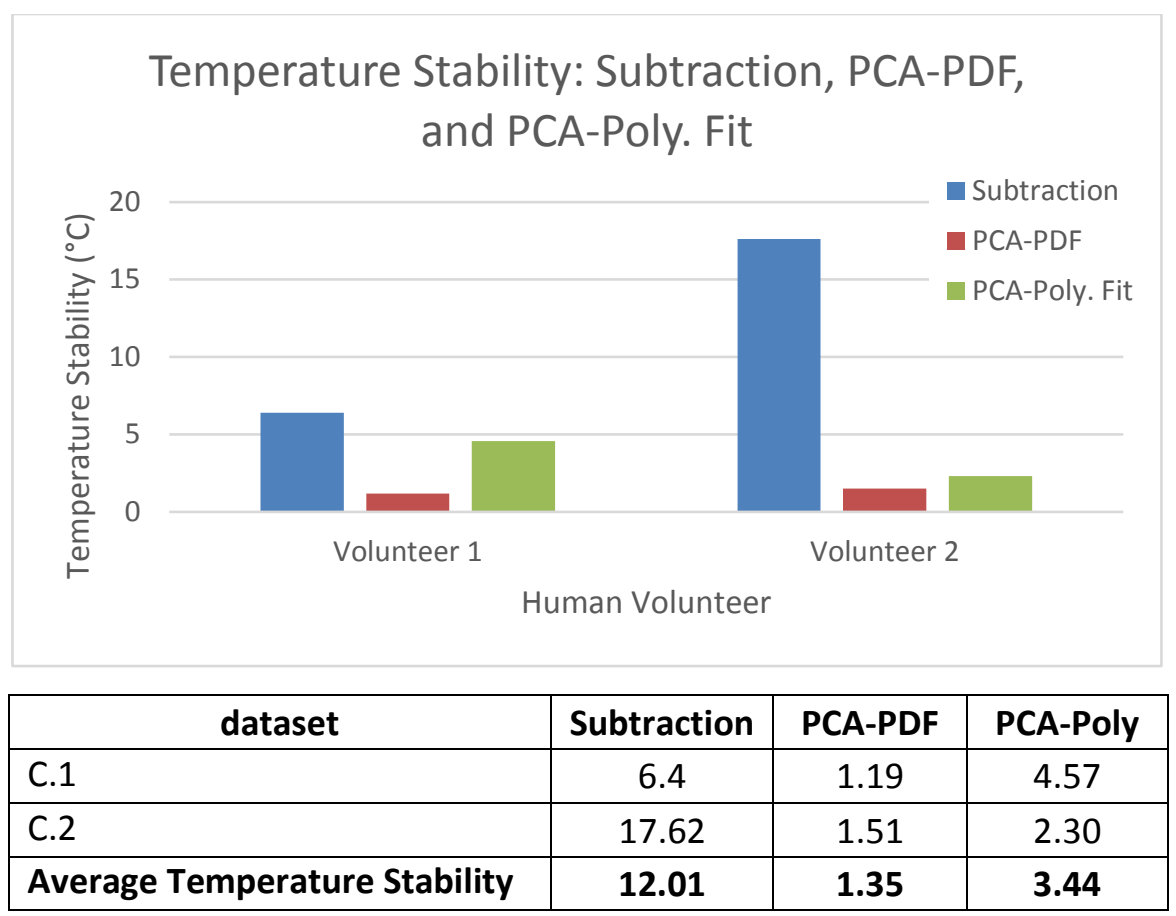

Figure 18: Human volunteers - temperature stability. This graph displays the temporal standard deviation of the spatial average of the temperature within a ROI for two human volunteer datasets.

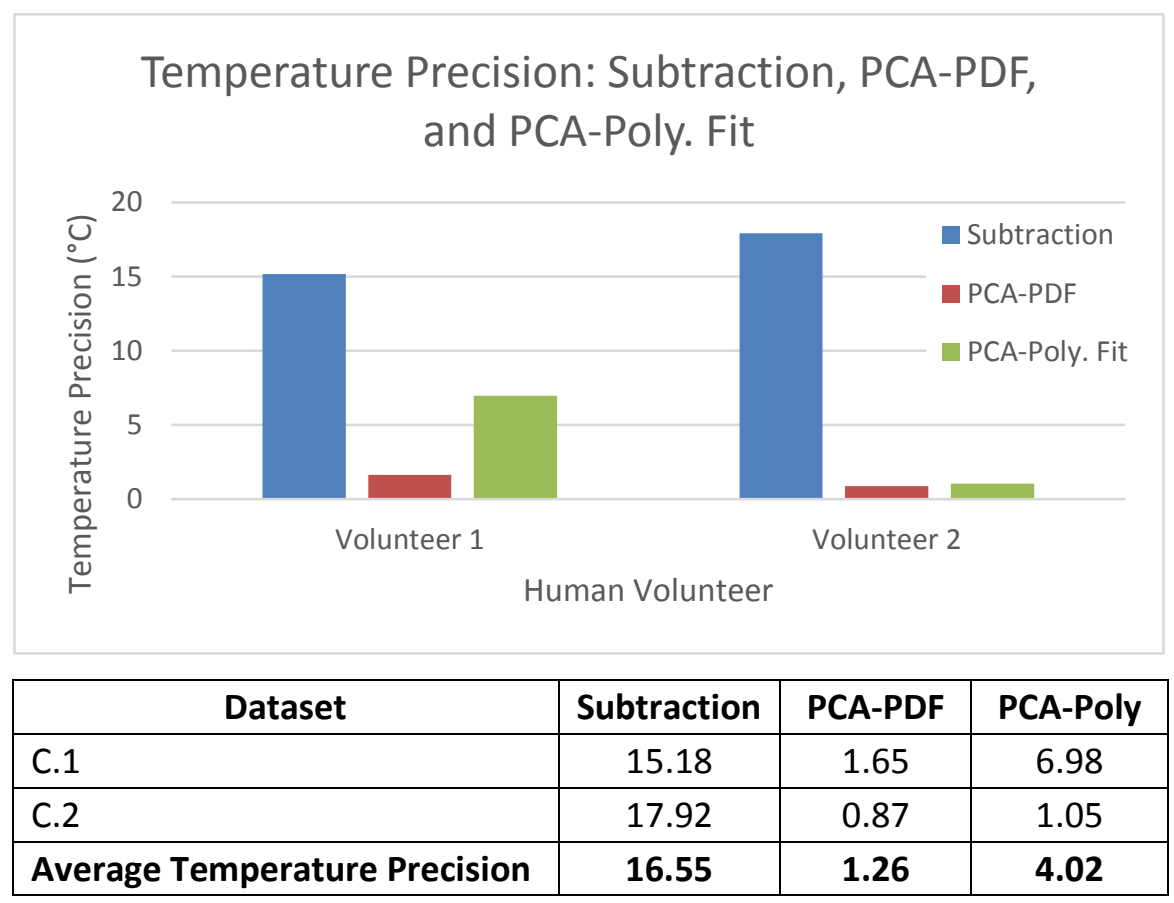

Figure 19: Human volunteers - temperature precision: this graph reports the temporal average of the spatial standard deviation of the temperature within an ROI for two human volunteer datasets. 
Across all porcine and human subjects analyzed, PCA-PDF achieves an average temperature stability and precision of $0.85^{\circ} \mathrm{C}$ and $1.00{ }^{\circ} \mathrm{C}$, respectively. For the same data, PCA-Polynomial Fit scores $1.97{ }^{\circ} \mathrm{C}$ and $2.22{ }^{\circ} \mathrm{C}$, respectively. PCA-PDF demonstrates superior correction to the state of the art PCA-Polynomial Fit benchmark. The first human volunteer dataset is selected for closer analysis and simulated heating.

Temperature standard deviation is displayed in Figure 20 for volunteer 1. PCA-PDF is compared with non-cumulative subtraction. PCA-PDF dramatically improves the temperature standard deviation in both inner regions and near to tissue boundaries.
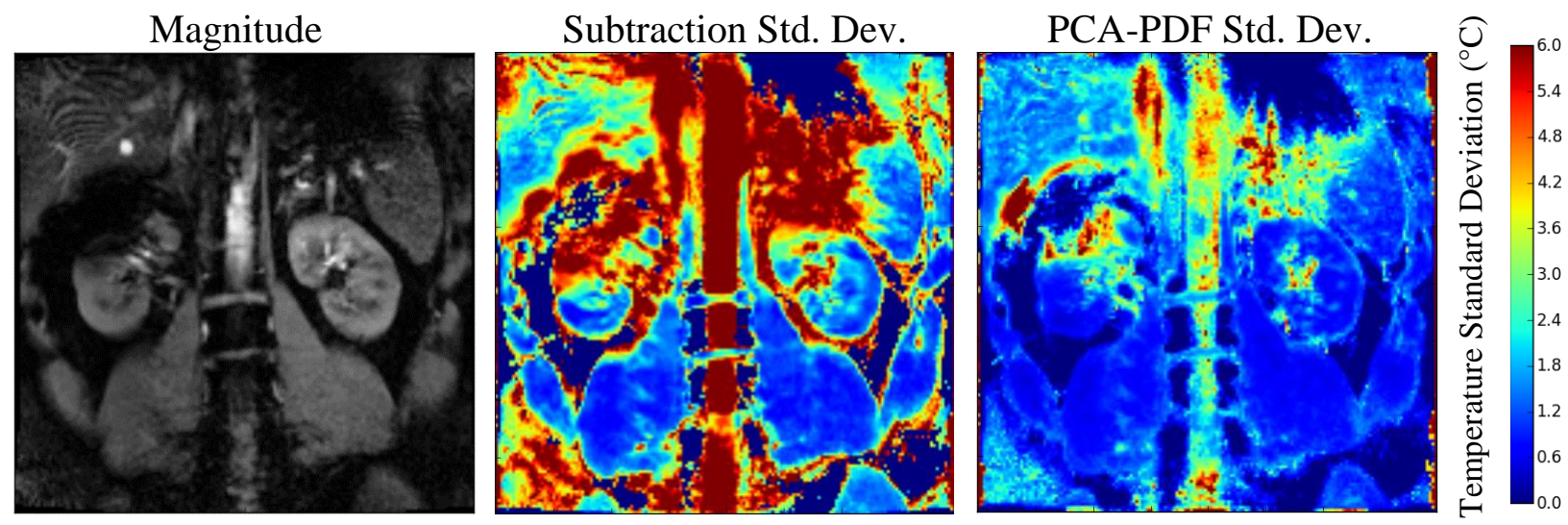

Figure 20: Temperature standard deviation maps: (left) magnitude image for reference, (center) noncumulative subtraction, (right) PCA-PDF. The combination of PCA and PDF improves temperature standard deviation at the tissue edge and the inner regions.

The following graphs depict a more detailed analysis of the spatial characteristics of the artifacts and corrective approximations. Figure 21 displays a 1D plot of the artifact profile along the red line in the image. The white and black arrows give the orientation of the plot. The polynomial fit method attempts to approximate the artifact, but is only capable of modeling low spatial frequency components. As such, the artifact in the image is largely unchanged by polynomial fit correction. 

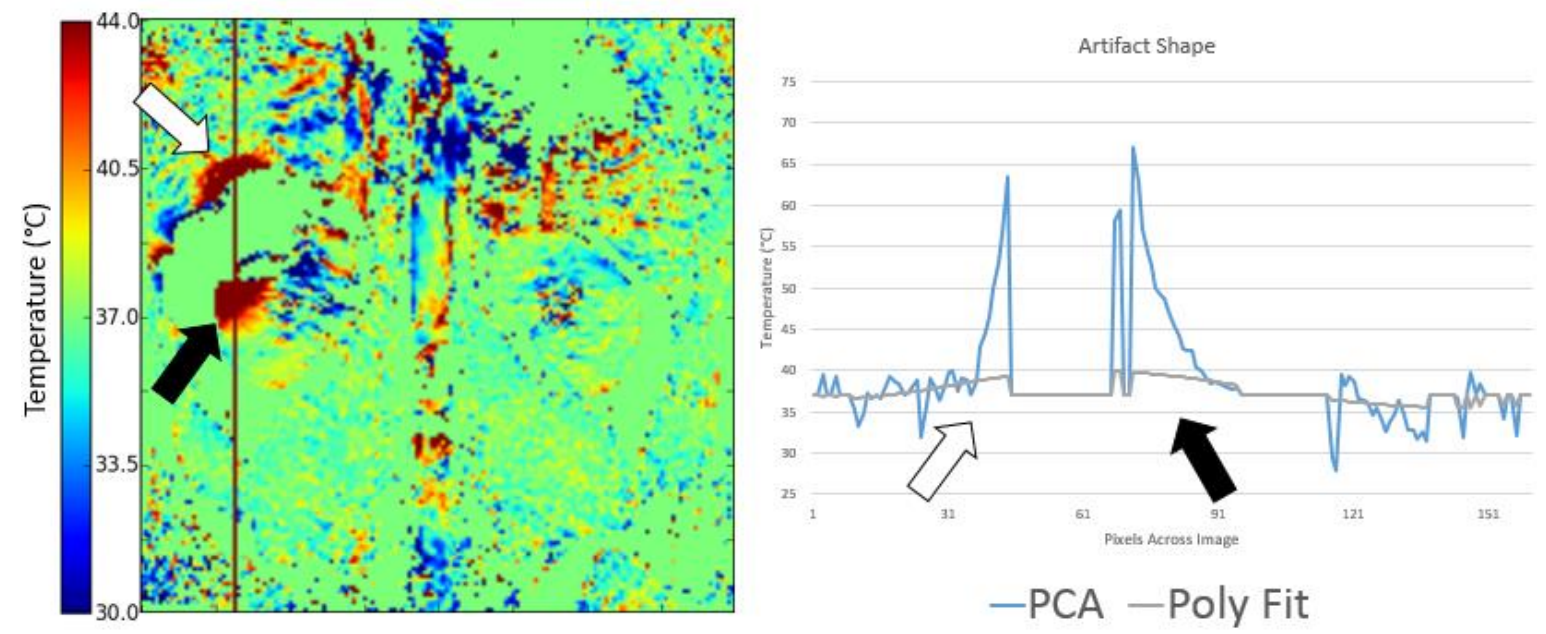

Figure 21: Spatial plot of artifact and polynomial fit correction. PCA (blue) depicts the artifacts remaining after PCA. Polynomial fit (grey) is only able to model the low spatial frequency component. Black and white arrows provide the orientation of the plot.

Figure 22 displays the PDF approximation of the artifact which agrees well with the artifact profile.
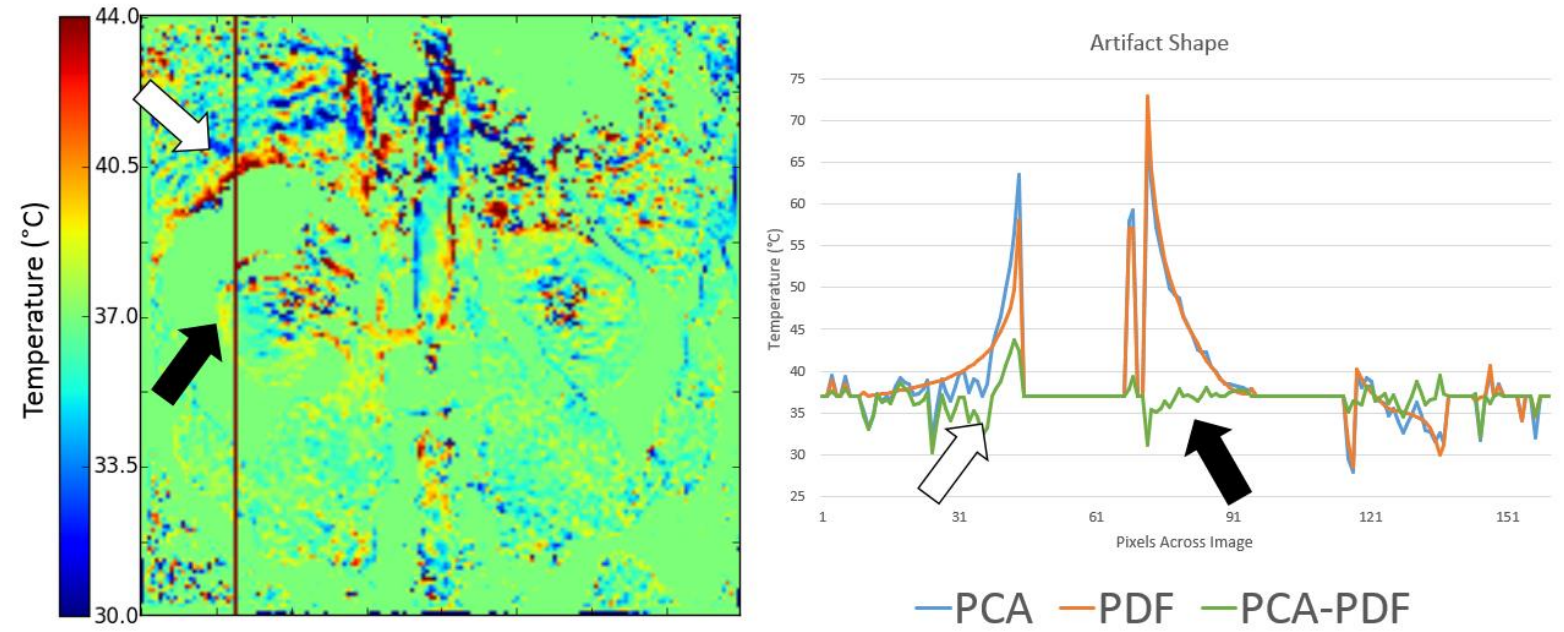

Figure 22: Spatial plot of artifact and PCA-PDF correction. PDF (orange) is able to fit the high and low spatial frequency components of the artifacts (PCA, blue). The combined PCA-PDF correction (green) displays a substantial improvement toward the baseline temperature $\left(37^{\circ} \mathrm{C}\right)$.

More importantly, Figure 23 displays that the injection of a hotspot causes virtually no change to the PDF response. This demonstrates that the PDF dipole model provides a good fit for artifacts 
and a poor fit for internal heating. This gives PDF the ability to discriminate between background susceptibility effects and internal heating even when they are overlapping.
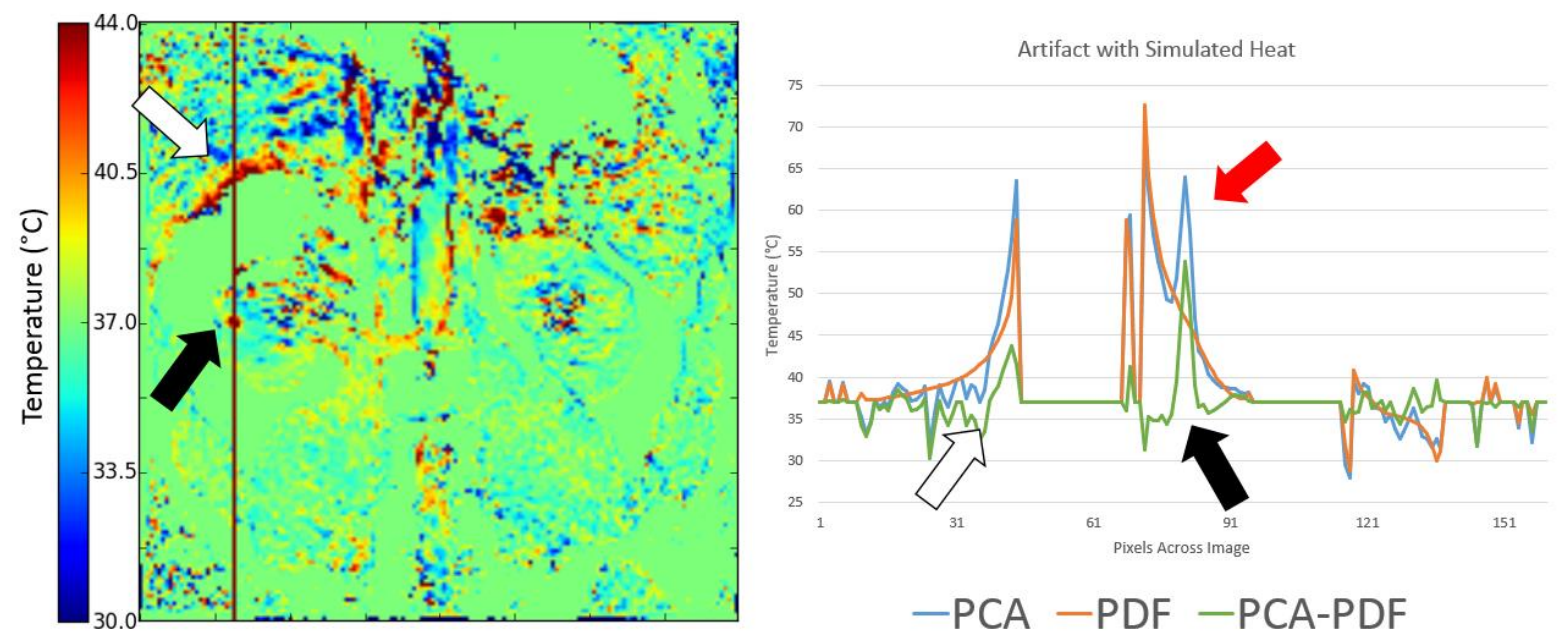

Figure 23: Injected hotspot and PCA-PDF correction. PCA (blue) displays overlapping artifact and hotspot (red arrow). PDF (orange) is able to model the artifact with minimal influence from the injected hotspot (red arrow). Resulting PCA-PDF correction (green) gives clean separation between artifact and hotspot.

Figure 24 displays the resulting error and also overlays the magnitude image. In this image the hotspot is only about 4 pixels away horizontally from the tissue boundary. The error plot displays the difference between PCA-PDF correction and ground truth. It indicates that PDF is not very accurate directly at the tissue border, but the error at the hotspot is roughly under $2.5^{\circ} \mathrm{C}$. 

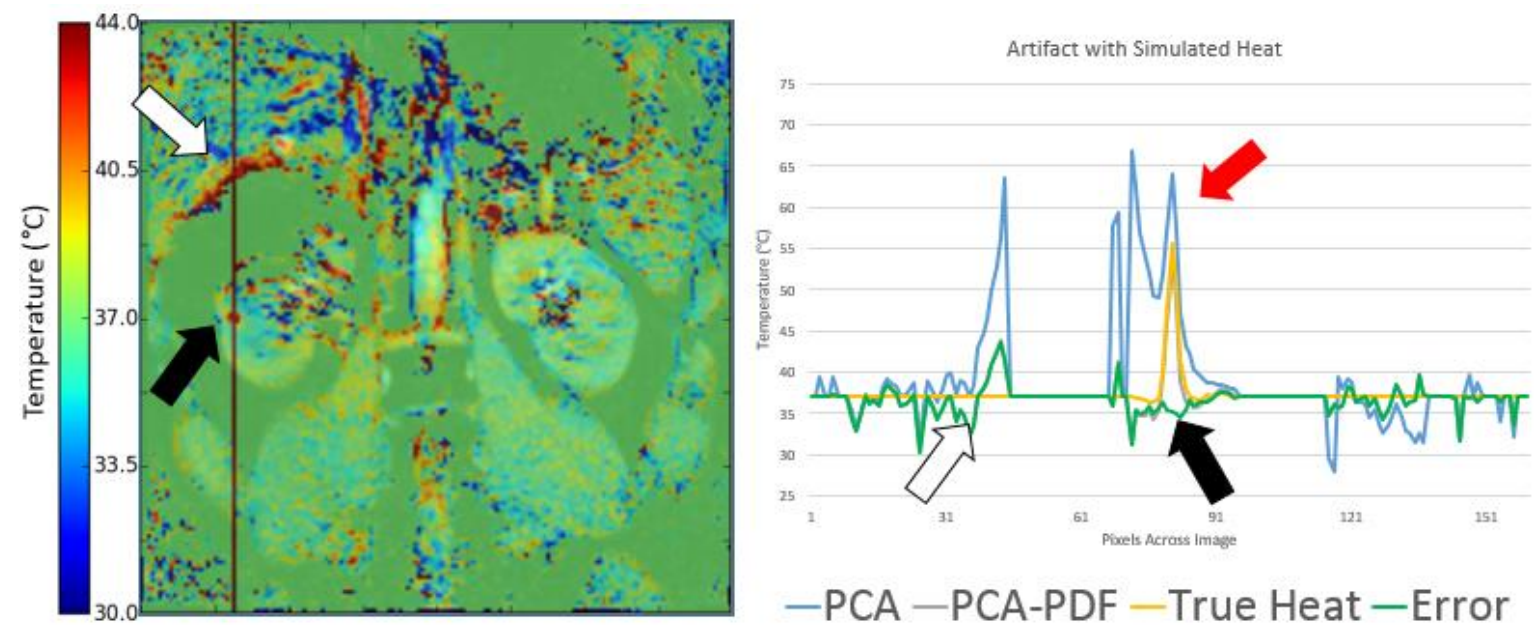

Figure 24: Injected hotspot with PCA-PDF error evaluation. This plot compares the PCA-PDF correction (grey) with the total injected heat (true heat, yellow). Their close match produces very little error (green). Also, the magnitude image is overlaid on the left to display the proximity of the hotspot to the edge of the tissue.

This level of accuracy can be compared with the average theoretical limit of the temperature uncertainty (Figure 25). In the hotspot zone, the average theoretical temperature uncertainty is approximately $1.07^{\circ} \mathrm{C}$.

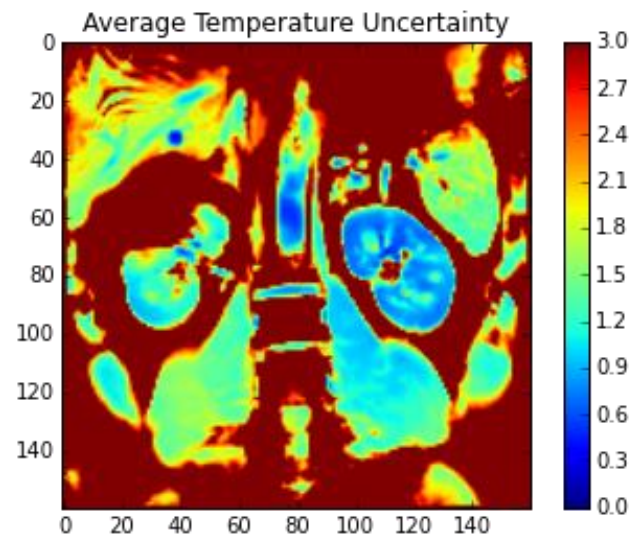

Figure 25: Average theoretical temperature uncertainty. This map displays the theoretical limit of the thermometry accuracy based on the SNR.

Note that theoretical temperature uncertainty is calculated from the SNR as

$$
\sigma(T)=\frac{1}{\gamma \alpha B_{O} T E} \frac{1}{S N R}[6][23](3.16)
$$


Where $\gamma$ is the gyromagnetic ratio, $\alpha$ is the proton resonance frequency change coefficient, $B_{o}$ is the magnetic field strength, and $T E$ is the echo time. This theoretical limit is most often compared with the temperature standard deviation, or temperature stability, which for PCA-PDF is $1.19^{\circ} \mathrm{C}$ and for PCA-Polynomial Fit is $4.57{ }^{\circ} \mathrm{C}$ in this dataset.

Figure 26 displays the comprehensive correction achieved with PCA-PDF. Together, PCA-PDF reveals a clear hotspot which would have been otherwise completely occluded by artifacts.

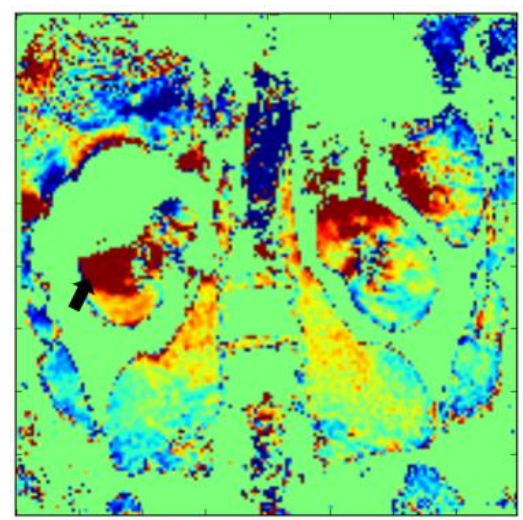

Subtraction

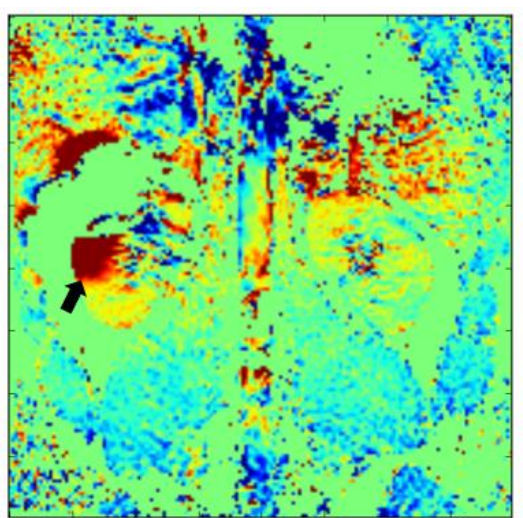

PCA

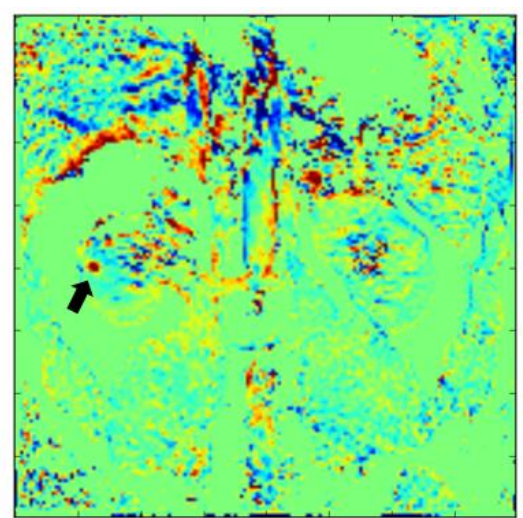

PCA-PDF

Figure 26: Progressive correction of artifacts with PCA and subsequently PDF. PCA and PDF together can correct the periodic and aperiodic artifacts. A hotspot (black arrow) obscured by artifacts can be clearly revealed through the PCA-PDF method.

\subsection{PDF Discussion}

These results demonstrate that PDF is able to accurately approximate the background susceptibility artifacts. In contrast, the polynomial fit is only able to match low spatial frequency components of artifacts. Most importantly, the model does not fit internal, heat-induced phase changes. This gives the algorithm a way of distinguishing susceptibility artifacts from heating. This is a crucial improvement over other referenceless methods which rely solely on spatial frequency to discern artifacts from heating, such as polynomial fit.

There are other methods, such as the near-harmonic model, which exploit the physical properties of the phase and improve the algorithm's ability to distinguish artifacts from heating [24]. However, this method is limited to regions that have homogeneous or linearly-changing susceptibility. Even more restrictive is the dependence of this algorithm on a carefully user- 
defined frame around the ROI. This is very difficult to perform reliably on moving tissue targets. The PDF method does not require any user interaction which dramatically increases its applicability.

Another major advantage over ROI-based methods is that PDF is applied across the entire FOV. This gives health care providers a more complete view of the surrounding tissue. This can be particularly crucial in abdominal applications where bone or air pockets can alter the trajectory of the HIFU beam in unpredictable ways.

PCA-PDF achieves an average temperature stability and precision of $0.85{ }^{\circ} \mathrm{C}$ and $1.00{ }^{\circ} \mathrm{C}$, respectively, in selected ROI's across all datasets. This is a substantial improvement over the state of the art PCA-Polynomial Fit benchmark which achieved an average temperature stability and precision of $1.97{ }^{\circ} \mathrm{C}$ and $2.22{ }^{\circ} \mathrm{C}$ respectively for the same data. Unfortunately, a standard deviation below $1{ }^{\circ} \mathrm{C}$ still does not guarantee that the temperature will always be accurate to within $\pm 1^{\circ} \mathrm{C}$. In the injected heat data, the accuracy is within $2.5^{\circ} \mathrm{C}$ in the hotspot region and even less accurate at the tissue border. It is very difficult to ensure accuracy within $\pm 1{ }^{\circ} \mathrm{C}$ at all times, however PCA-PDF helps bring the temperature stability, $1.19{ }^{\circ} \mathrm{C}$, close to the theoretical limit of the temperature uncertainty, $1.07^{\circ} \mathrm{C}$, for the selected kidney. In comparison the benchmark scored $4.57^{\circ} \mathrm{C}$ for the temperature stability in the same ROI.

One of the drawbacks of this PDF method is its use of a 2D dipole model which can fail to accurately model the true 3D reality. This can be seen in PDF's failure to remove the artifacts in the center of the tissue in the $3^{\text {rd }}$ (A.2 right) kidney motion dataset. However, without the additional 3D phase information, it is extremely difficult to ascertain whether the phase changes are truly artifacts or low spatial frequency heating. Unfortunately it is not possible to acquire a coherent 3D volume without intra-dynamic motion while maintaining a high frame rate. This will likely become possible with future developments in acquisition methods. Until then, the 2D dipole model provides a very close fit for the majority of artifacts presented in the data.

Another limitation of PDF is its performance directly at the edge of tissue borders. PDF is reported to have excellent separation between local and background dipole effects for all regions except within 5 pixels of the tissue boundary [29]. The injected heat data was placed only 4 pixels from the edge of the tissue. Despite the close proximity, PDF was able to separate the background susceptibility artifact and clearly reveal the hotspot. 
As mentioned in the PCA chapter, the processing time is another concern. PCA was able to reduce average computation time from $152 \mathrm{~ms}$ to $26 \mathrm{~ms}$. The average processing time for PDF is about $147 \mathrm{~ms}$ and the overhead of the application is about $81 \mathrm{~ms}$. As such, without the PCA speed improvement, the total computation time is about $353 \mathrm{~ms}$ on average, still well under the dynamic scan time for a single image $573 \mathrm{~ms}$. 


\section{Chapter 4 \\ Thesis Summary and Conclusion}

\subsection{Thesis Summary}

The combination of PCA and PDF provides comprehensive correction of both periodic and aperiodic artifacts. The performance of PCA-PDF has been tested in porcine and human kidneys as well as porcine head and neck regions, with superior correction to benchmark methods. Note that this design of PCA-PDF is not application specific. This means that it has potential to be applied to a range of clinical needs; however, each use case will require further validation with data specific to the anatomy. This also means that there is potential for PCA-PDF to be further optimized for different cases. PCA is very versatile because it does not rely on any a priori knowledge of predefined features; instead it forms eigenimages based on the statistical characteristics of the data it is given. In contrast, PDF is inherently linked to the physical structure of the anatomy that it attempts to model dipoles around. Indeed, PDF's ability is limited by the background region it has to operate within. This background region is automatically defined using SNR and thus requires low signal regions to form a background region. As such, areas of tightly packed, high signal tissue may limit PDF's corrective ability. In such cases, it may be possible to create an option for user defined background regions or for intelligent switching to alternative algorithms, such as polynomial fit, which may perform better in such instances. It may also be possible to exploit the data from orthogonal slices to gain more information on the 3D susceptibility environment. This is challenging and may require the acquisition of pre-operative 3D scans and the use of organ-specific template models that can be fitted to the data and provide a rough estimate of the missing 3D information during a procedure. As such, PDF has potential for further modification for different clinical applications.

Nevertheless, the current form of PCA-PDF achieves superior correction compared to benchmarks. PCA demonstrates effective correction of periodic artifacts while reducing computational costs. PDF provides a correction of aperiodic artifacts with excellent discrimination between local heating and edge susceptibility artifacts. While the accuracy is not guaranteed to be within $\pm 1{ }^{\circ} \mathrm{C}$ at all times, PDF's temperature stability and precision, of $0.85{ }^{\circ} \mathrm{C}$ and $1.00{ }^{\circ} \mathrm{C}$, respectively, demonstrate a statistical improvement compared with the state of the art hybrid method benchmark which scored $1.97{ }^{\circ} \mathrm{C}$ and $2.22{ }^{\circ} \mathrm{C}$, respectively. Indeed it is very 
difficult to guarantee $\pm 1{ }^{\circ} \mathrm{C}$ accuracy, but PCA and PDF provide advantages to help increase the probability of accurate thermometry. PCA lowers computational costs and allows for long observation periods to increase the likelihood of a close matching reference image. Meanwhile, PDF attempts to model all residual edge susceptibility artifacts which fit the physical magnetic dipole model. The goals of this thesis were to develop an artifact correction algorithm A) to correct thermometry to an accuracy of $\pm 1{ }^{\circ} \mathrm{C}$ within the targeted cell and to $\pm 3{ }^{\circ} \mathrm{C}$ in the periphery, and B) having a processing time, for a single dynamic, less than the dynamic scan time of the MR sequence. Objective (A) cannot be strictly met for every dynamic image. However, PCA-PDF has achieved a substantial improvement toward this goal in abdominal datasets with challenging artifacts. Also, PCA-PDF achieves notable improvement over the state of the art. Objective (B) is completely satisfied and provides a framework that accommodates the demands of future acquisition strategies for rapid 3D imaging. As such, PCA-PDF provides statistically accurate thermometry at a speed that is feasible for real-time applications.

\subsection{Conclusion}

The PCA-PDF hybrid method provides accurate, real-time thermometry for abdominal applications. To further improve accuracy it may be possible to use multiple echoes for the PDF processing, as the original method typically uses a multi-echo acquisition. Also, having 3D datasets will facilitate the removal of more artifacts and may allow for a completely referenceless PDF method. 


\section{References}

[1] N. Papanikolaou et al., "Tissue Inhomogeneity Corrections for Megavoltage Photon Beams," Am. Assoc. Phys. Med., vol. 85, pp. 1-135, 2004.

[2] J. W. Jenne, T. Preusser, and M. Günther, "High-intensity focused ultrasound: Principles, therapy guidance, simulations and applications," Z. Med. Phys., vol. 22, pp. 311-322, 2012.

[3] A. Muller et al., "Management of respiratory motion in extracorporeal high-intensity focused ultrasound treatment in upper abdominal organs: current status and perspectives.," Cardiovasc. Intervent. Radiol., vol. 36, no. 6, pp. 1464-76, Dec. 2013.

[4] Canadian Cancer Society, "Canadian Cancer Statistics Special topic: Predictions of the future burden of cancer in Canada," Public Heal. Agency Canada, pp. 1-151, 2015.

[5] J. M. Maris, "Recent advances in neuroblastoma.," N. Engl. J. Med., vol. 362, no. 23, pp. 2202-11, Jun. 2010.

[6] V. Rieke and K. B. Pauly, "MR thermometry," J. Magn. Reson. Imaging, vol. 27, no. 2, pp. 376-390, 2008.

[7] D. W. McRobbie et al., MRI From Picture to Proton. New York: Cambridge University Press, 2006.

[8] A. D. Elster and J. H. Burdette, Questions and Answers in Magnetic Resonance Imaging. St. Louis: Mosby, 2001.

[9] E. M. Haacke et al., Magnetic Resonance Imaging: Physical Principles and Sequence Design. Hoboken, NJ: John Wiley \& Sons, Inc., 2014.

[10] Y. Ishihara et al., "A precise and fast temperature mapping using water proton chemical shift.," Magn Reson Med, vol. 34, no. 6, pp. 814-823, 1995.

[11] O. I. Craciunescu et al., "Accuracy of real time noninvasive temperature measurements using magnetic resonance thermal imaging in patients treated for high grade extremity soft tissue sarcomas," Med. Phys., vol. 36, no. 11, p. 4848, 2009. 
[12] T. Ta and T. M. Porter, "Thermosensitive liposomes for localized delivery and triggered release of chemotherapy," J. Control. Release, vol. 169, no. 1-2, pp. 112-125, 2013.

[13] M. W. Dewhirst et al., "Thermal Dose Requirement for Tissue Effect: Experimental and Clinical Findings," Proc SPIE Int Soc Opt Eng, vol. 4954, no. 37, pp. 1-35, 2003.

[14] F. Maier et al., "Velocity navigator for motion compensated thermometry.," MAGMA, vol. 25, no. 1, pp. 15-22, Feb. 2012.

[15] V. Auboiroux et al., "Respiratory-gated MRgHIFU in upper abdomen using an MRcompatible in-bore digital camera.," Biomed Res. Int., vol. 2014, p. 421726, Jan. 2014.

[16] V. Auboiroux et al., "Ultrasonography-based 2D motion-compensated HIFU sonication integrated with reference-free MR temperature monitoring: a feasibility study ex vivo.," Phys. Med. Biol., vol. 57, no. 10, pp. N159-71, 2012.

[17] K. K. Vigen et al., "Triggered, Navigated, Multi-Baseline Method for Proton Resonance Frequency Temperature Mapping With Respiratory Motion,” Magn. Reson. Med., vol. 50, no. 5, pp. 1003-1010, 2003.

[18] B. D. de Senneville, C. Mougenot, and C. T. W. Moonen, "Real-time adaptive methods for treatment of mobile organs by MRI-controlled high-intensity focused ultrasound.," Magn. Reson. Med., vol. 57, no. 2, pp. 319-30, Feb. 2007.

[19] S. Pichardo et al., "In vivo optimisation study for multi-baseline MR-based thermometry in the context of hyperthermia using MR-guided high intensity focused ultrasound for head and neck applications," Int. J. Hyperth., vol. 30, no. 8, pp. 579-592, Dec. 2014.

[20] G. Maclair et al., "PCA-Based Magnetic Field Modeling : Application for On-Line MR Temperature Monitoring," in Medical Image Computing and Computer-Assisted Intervention - MICCAI 2007, Berlin, Heidelberg: Springer Berlin Heidelberg, 2007, pp. 411-419.

[21] B. D. de Senneville et al., "MR-guided thermotherapy of abdominal organs using a robust PCA-based motion descriptor.," IEEE Trans. Med. Imaging, vol. 30, no. 11, pp. 1987-95, Nov. 2011. 
[22] W. a Grissom et al., "Hybrid referenceless and multibaseline subtraction MR thermometry for monitoring thermal therapies in moving organs.," Med. Phys., vol. 37, no. 9, pp. 50145026, 2010.

[23] V. Rieke et al., "Referenceless PRF shift thermometry.," Magn. Reson. Med., vol. 51, no. 6, pp. 1223-31, Jun. 2004.

[24] R. Salomir et al., "Reference-free PRFS MR-thermometry using near-harmonic 2-D reconstruction of the background phase," IEEE Trans. Med. Imaging, vol. 31, no. 2, pp. 287-301, 2012.

[25] W. a. Grissom et al., "Reweighted $\ell 1$ referenceless PRF shift thermometry," Magn. Reson. Med., vol. 64, no. 4, pp. 1068-1077, Jun. 2010.

[26] B. D. de Senneville et al., "Motion correction in MR thermometry of abdominal organs: a comparison of the referenceless vs. the multibaseline approach.," Magn. Reson. Med., vol. 64, no. 5, pp. 1373-81, Nov. 2010.

[27] M. Turk and A. Pentland, "Eigenfaces for Recognition," Journal of Cognitive Neuroscience, vol. 3. pp. 71-86, 1991.

[28] S. Roujol et al., "Real-time MR-thermometry and dosimetry for interventional guidance on abdominal organs.," Magn. Reson. Med., vol. 63, no. 4, pp. 1080-7, Apr. 2010.

[29] T. Liu et al., "A novel background field removal method for MRI using projection onto dipole fields (PDF)," NMR Biomed., vol. 24, no. 9, pp. 1129-1136, 2011.

[30] R. Bhagwandien et al., "Numerical analysis of the magnetic field for arbitrary magnetic susceptibility distributions in 2D," Magn. Reson. Imaging, vol. 10, no. 2, pp. 299-313, 1992.

[31] C. J. G. Bakker et al., "Susceptibility artifacts in 2DFT spin-echo and gradient-echo imaging: The cylinder model revisited," Magn. Reson. Imaging, vol. 11, no. 4, pp. 539$548,1993$.

[32] J. Escoff and A. Bouakaz, "Therapeutic Ultrasound," Adv. Exp. Med. Biol., vol. 880, pp. 
1-465, 2016. 


\section{Appendices}

\section{Appendix A. Additional Standard Deviation Maps from PCA Results}

\begin{tabular}{|l|r|r|r|r|}
\hline Dataset & \multicolumn{4}{|c|}{ A.1 Right Kidney } \\
\hline Initial Number of Images in Atlas & 0 & 50 & 50 & 12 \\
\hline Final Number of Images in Atlas & 0 & 50 & 12 & 12 \\
\hline Average Computation Time $(\mathbf{m s})$ & 0 & 150 & 36 & 35 \\
\hline Standard Deviation in Kidney $\left({ }^{\circ} \mathrm{C}\right)$ & 1.17 & 0.35 & 0.43 & 0.62 \\
\hline
\end{tabular}
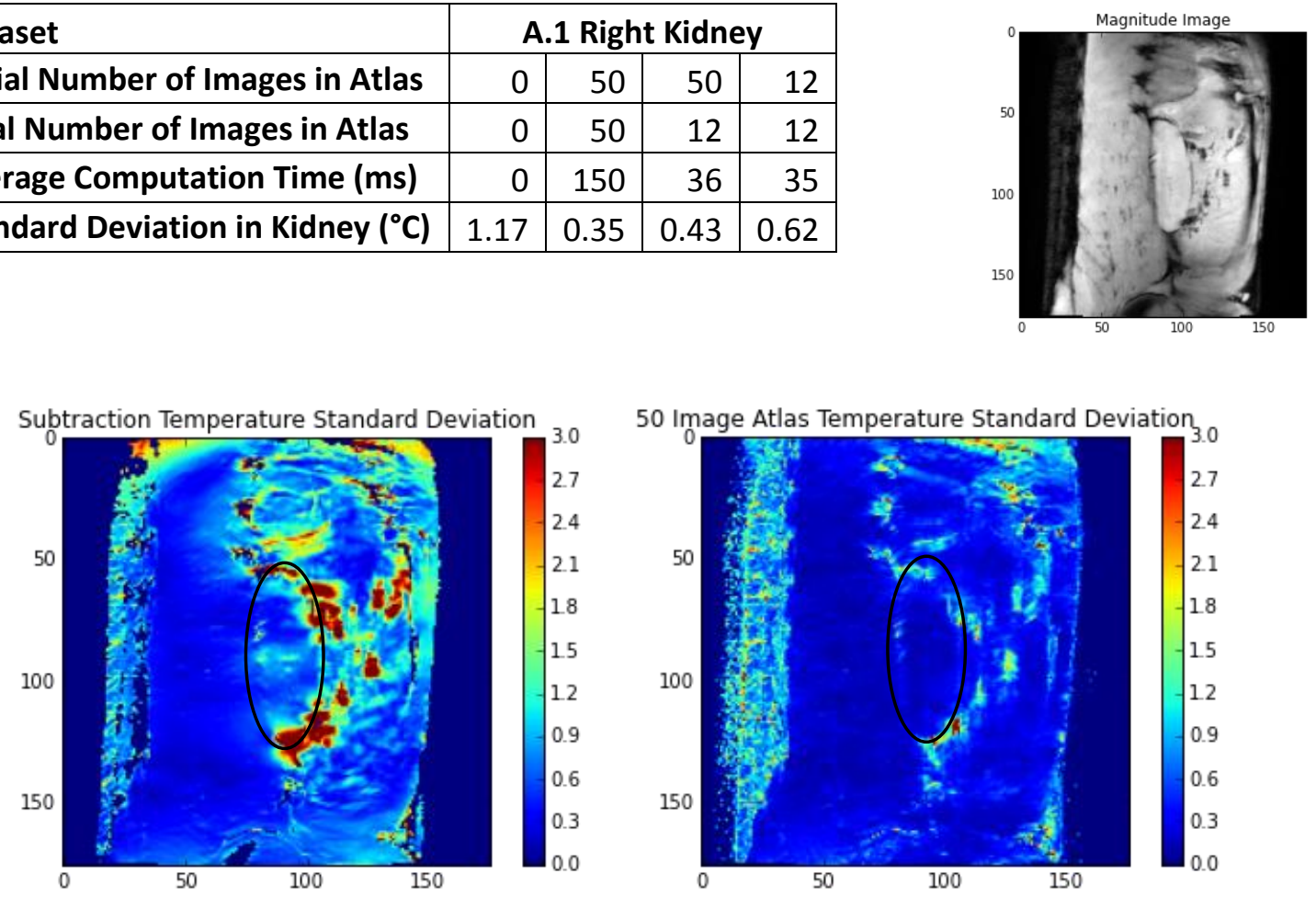

12(50) Eigenimage Atlas Temperature Standard Deviation
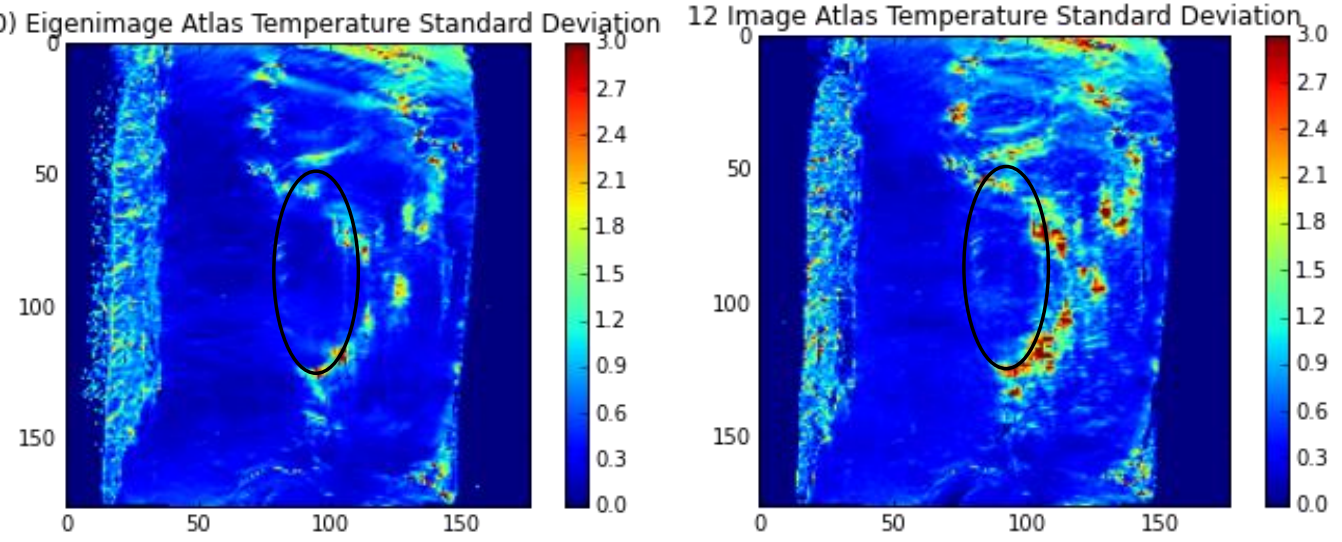

Figure 27: Standard deviation maps for A.1 right kidney motion dataset: Table indicates number of dynamics used in the atlas, computation time, and standard deviation in the kidney for each processing method. 


\begin{tabular}{|l|r|r|r|r|}
\hline Dataset & \multicolumn{4}{|c|}{ A.2 Left Kidney } \\
\hline Initial Number of Images in Atlas & 0 & 50 & 50 & 6 \\
\hline Final Number of Images in Atlas & 0 & 50 & 6 & 6 \\
\hline Average Computation Time $(\mathrm{ms})$ & 0 & 155 & 21 & 21 \\
\hline Standard Deviation in Kidney $\left({ }^{\circ} \mathrm{C}\right)$ & 1.61 & 0.46 & 0.59 & 0.68 \\
\hline
\end{tabular}
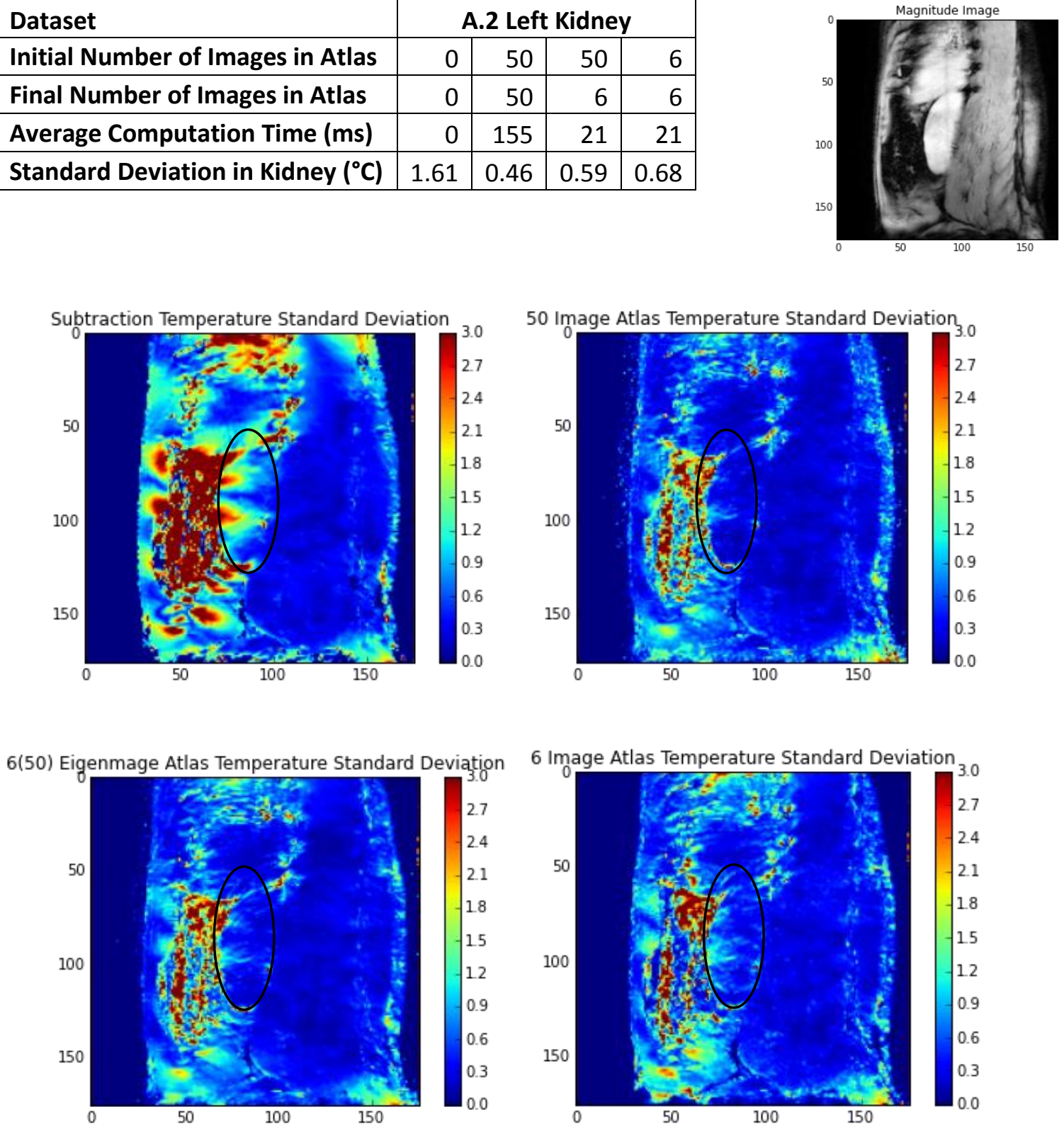

Figure 28: Standard deviation maps for A.2 left kidney motion dataset: Table indicates number of dynamics used in the atlas, computation time, and standard deviation in the kidney for each processing method. 


\begin{tabular}{|l|r|r|r|r|}
\hline Dataset & \multicolumn{4}{|c|}{ A.2 Right Kidney } \\
\hline Initial Number of Images in Atlas & 0 & 50 & 50 & 6 \\
\hline Final Number of Images in Atlas & 0 & 50 & 6 & 6 \\
\hline Average Computation Time $(\mathbf{m s})$ & 0 & 152 & 22 & 21 \\
\hline Standard Deviation in Kidney $\left({ }^{\circ} \mathrm{C}\right)$ & 0.95 & 0.40 & 0.46 & 0.84 \\
\hline
\end{tabular}
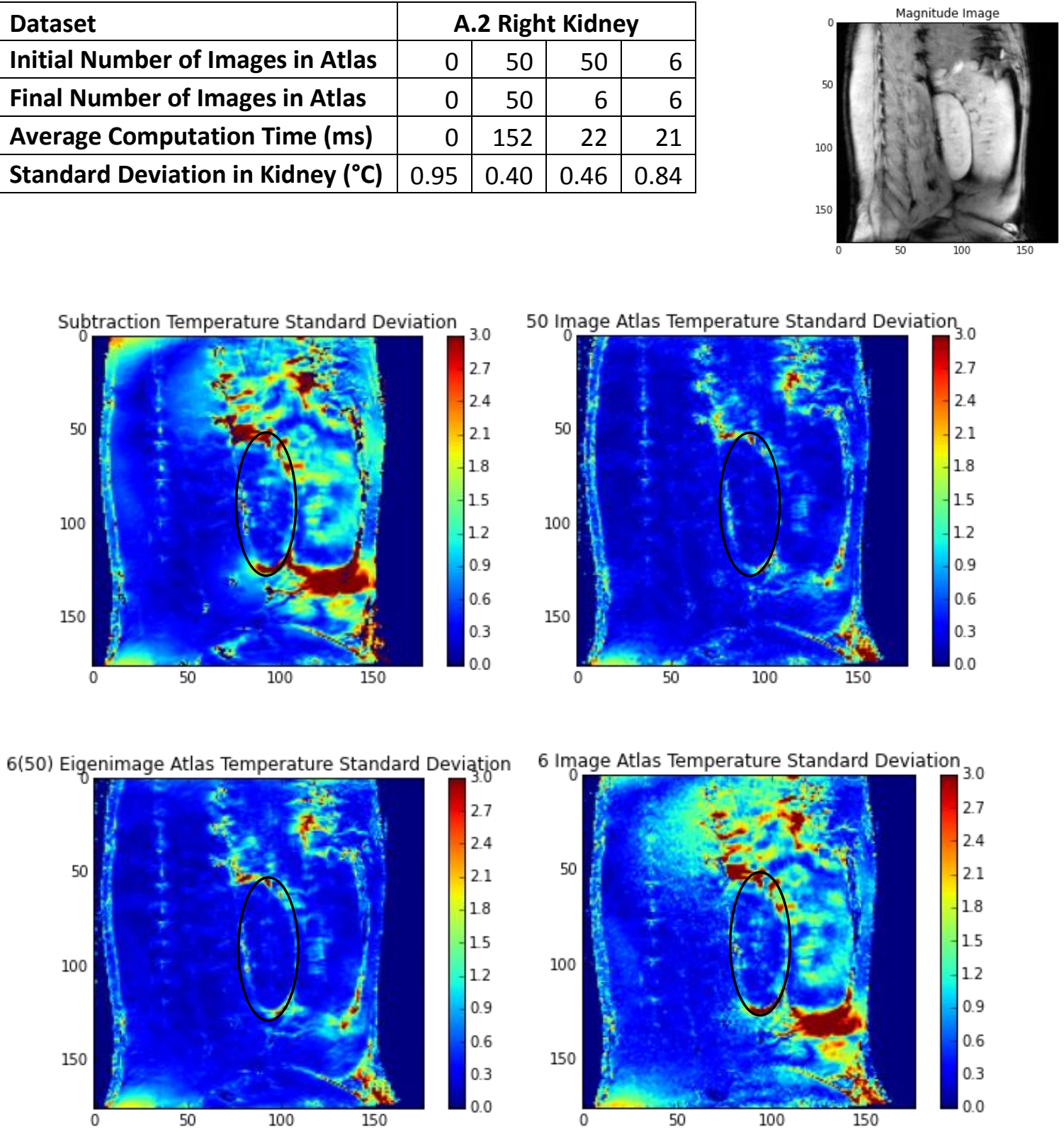

Figure 29: Standard deviation maps for A.2 right kidney motion dataset: Table indicates number of dynamics used in the atlas, computation time, and standard deviation in the kidney for each processing method. 


\begin{tabular}{|l|r|r|r|r|}
\hline Dataset & \multicolumn{4}{|c|}{ A.3 Left Kidney } \\
\hline Initial Number of Images in Atlas & 0 & 50 & 50 & 13 \\
\hline Final Number of Images in Atlas & 0 & 50 & 13 & 13 \\
\hline Average Computation Time $(\mathbf{m s})$ & 0 & 150 & 38 & 37 \\
\hline Standard Deviation in Kidney $\left({ }^{\circ} \mathbf{C}\right)$ & 11.40 & 0.53 & 0.70 & 0.80 \\
\hline
\end{tabular}
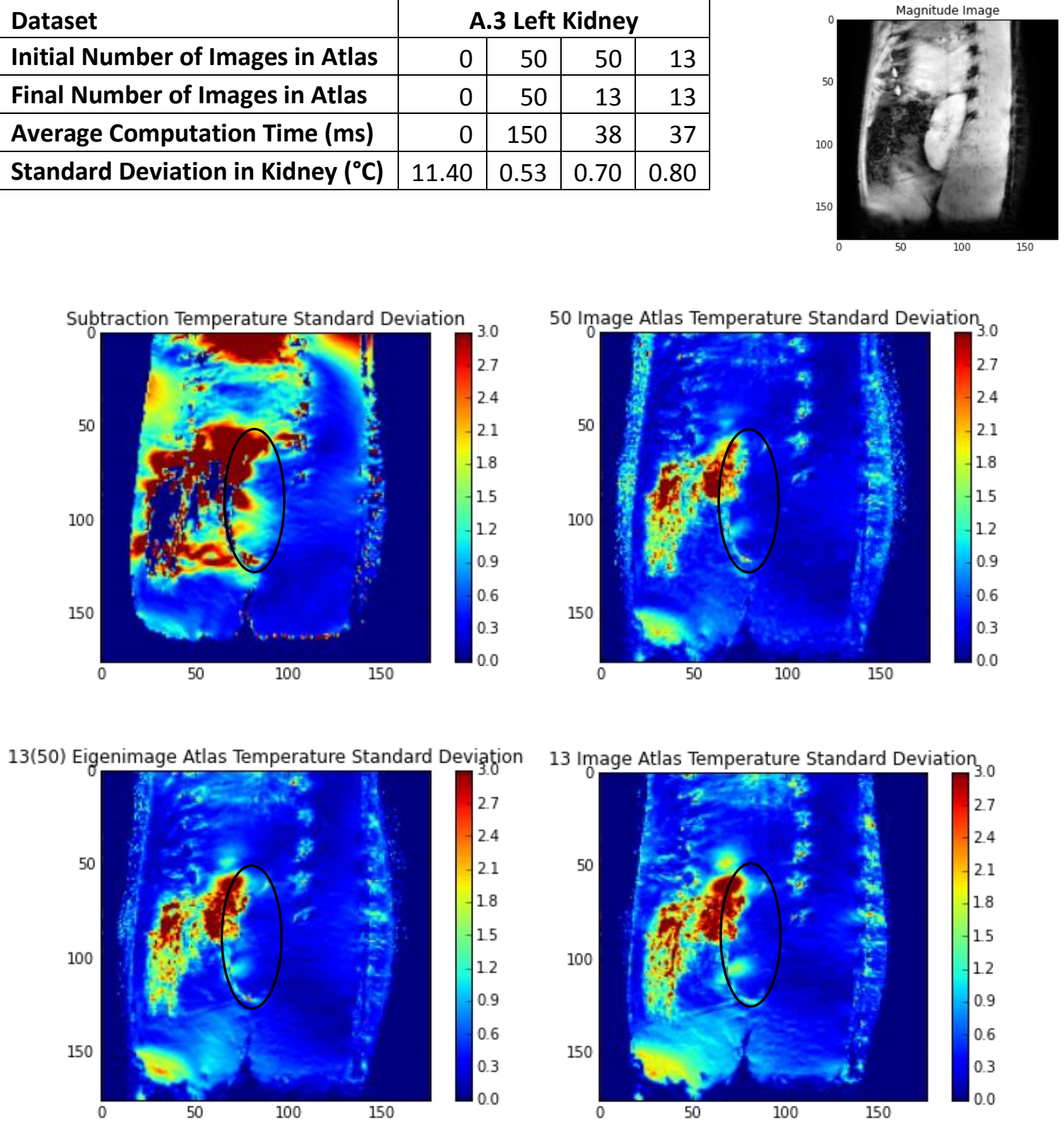

Figure 30: Standard deviation maps for A.3 left kidney motion dataset: Table indicates number of dynamics used in the atlas, computation time, and standard deviation in the kidney for each processing method. 


\begin{tabular}{|l|r|r|r|r|}
\hline Dataset & \multicolumn{4}{|c|}{ A.3 Right Kidney } \\
\hline Initial Number of Images in Atlas & 0 & 50 & 50 & 6 \\
\hline Final Number of Images in Atlas & 0 & 50 & 6 & 6 \\
\hline Average Computation Time $(\mathbf{m s})$ & 0 & 152 & 20 & 20 \\
\hline Standard Deviation in Kidney $\left({ }^{\circ} \mathbf{C}\right)$ & 0.71 & 0.26 & 0.30 & 0.41 \\
\hline
\end{tabular}
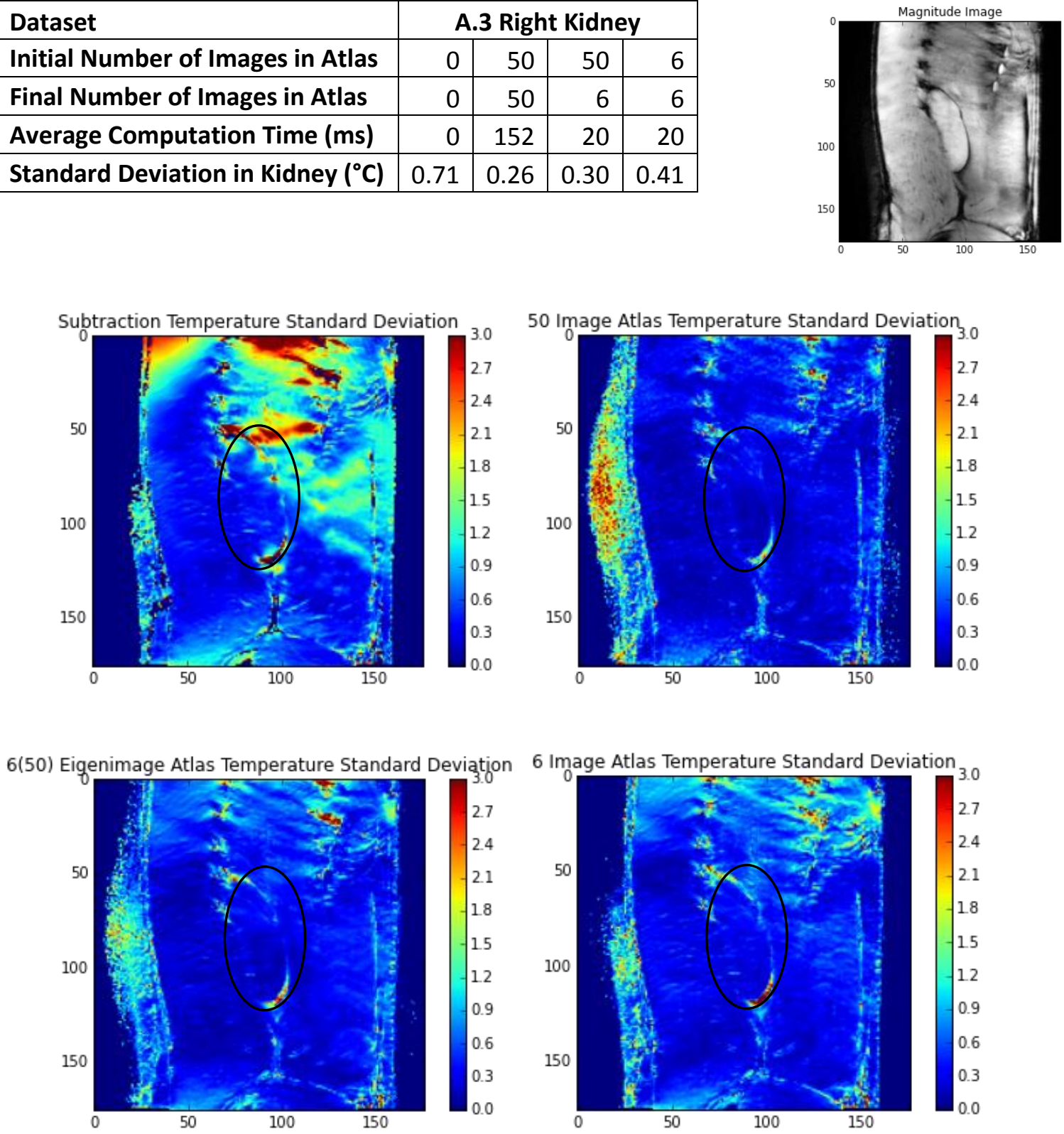

Figure 31: Standard deviation maps for A.3 right kidney motion dataset: Table indicates number of dynamics used in the atlas, computation time, and standard deviation in the kidney for each processing method. 